\title{
Mechanisms of Plasticity in Near-Theoretical Strength sub-100 nm Si Nanocubes
}

Andrew J. Wagner $\stackrel{\dagger}{-}$ Eric D. Hintsala ${ }^{\dagger}$, Prashant Kumar, William W. Gerberich, K. Andre Mkhoyan*

Andrew J. Wagner, Eric D. Hintsala, Prashant Kumar, William W. Gerberich, K. Andre Mkhoyan

Department of Chemical Engineering and Materials Science, University of Minnesota, Minneapolis, MN, 55455, USA

E-mail: $\underline{\text { mkhoyan@umn.edu }}$

ذ These authors contributed equally to this work.

Keywords: In situ transmission electron microscopy (TEM), mechanical properties, silicon, nanoparticles, plasticity

\begin{abstract}
Silicon is one of the most technologically important materials, used extensively in electronics, solar cells, micro-electro-mechanical systems (MEMS) based devices and more. Yet its mechanical properties are not well understood at the nanoscale where it is often utilized. Experimental measurements under a variety of loading conditions are needed, and compression experiments are particularly lacking. Here, the elastic-plastic response of $20 \mathrm{~nm}$ to $65 \mathrm{~nm}$ cubic Si nanocubes under uniaxial compression is investigated. The purely elastic limit of these nanocubes is observed to be up to 0.07 true strain at $7 \mathrm{GPa}$ true stress with an upper yield point of 0.20 true strain and $11 \mathrm{GPa}$ true stress. Investigation of the nature of dislocations generated during deformation of these nanocubes using post-mortem analysis in the TEM provides evidence that leading partial dislocations are the dominant source of plasticity at this scale.
\end{abstract}




\section{Introduction}

The changes in mechanical response of a diverse set of nanoscale materials compared to their bulk counterparts has created a new challenge for device design while opening doors to new applications [1-10]. In silicon, these changes result in a size-based ductile-to-brittle transition [11] and an increase of the elastic limit [11-17]. These effects are promising for a number of critical applications, including transistor optimization. Straining of the channel in silicon-based transistors offer improvements in electron and hole mobility with the potential to increase drive current by two to four times [18]. New device structures (e.g. FINFETs) continue to push these strain-induced improvements [19-21]. Therefore, the maximum achievable strain in these nanoscale crystalline structures without dislocation nucleation needs to be determined. To accomplish this, recent experimental innovations in transmission electron microscopy (TEM) holders have enabled in-situ TEM investigation of mechanical properties of defect-free nanoscale specimens.

The atomistic mechanisms contributing to elasticity and plasticity in silicon nanowires have been examined in-situ by tensile and bending tests in the TEM [12,22-26]. Under tension and bending conditions, a size-dependence on elastic limit and yielding has been observed in less than $100 \mathrm{~nm}$ in length Si nanowires [24,25]. Additionally, Stan et al. [26] demonstrated occurrence of a brittle fracture in sub-60 nm-thick Si nanowires at bending stresses of $17 \mathrm{GPa}$, although the nanowires were found to exhibit some plasticity. On the other hand, Wang et al. [23] reported observation of plasticity in sub-50 nm Si nanowires by bending and showed presence of both perfect and partial dislocation post-mortem. However, the origin of the mechanism of plasticity in sub-100 nm Si crystals is still unsettled. It should be noted that the challenge associated with in-situ TEM imaging of dislocations in nanowire during such bending experiments is that the orientation and defocus are changing at each step, making images less reliable. 
In addition to bending and tensile experiments, mechanisms of plastic deformation in sub-100 nm Si structures can be studied by compression experiments, which often closely resembles strain in devices. Additionally, compression testing is better suited for studying low aspect ratio specimens, which is closer to device dimensions than nanowires and experience less significant changes in orientation and focusing. In-situ TEM investigations of plasticity in sub-100 $\mathrm{nm}$ structures under compression have been largely limited by experimental challenges with stability of indenter-incorporated holders.

Molecular dynamics simulations performed for the $\mathrm{Si}$ nanostructures under compression at low temperature have predicted a variety of mechanisms to accommodate plasticity [27-32]: $\{111\}$ slip by perfect straight-segmented dislocation loops with burgers vector $b=\mathrm{a} / 2<\overline{1} 10>[27,28]$, the dissociation of these $\{111\}$ dislocation loops into two partial $b=\mathrm{a} / 6<11 \overline{2}>$ dislocations, $\{110\}$ slip by perfect dislocations loops with $b$ $=\mathrm{a} / 2<01 \overline{1}>$ [29], deformation twinning [30] and phase transformation [28,31].

Here we present the results of in-situ TEM compression experiments on sub-100 nm Si nanocubes performed with a high-stability indenter-incorporated holder. We present clear identification of the onset of dislocation nucleation and propagation, and characterization of plasticity mechanisms.

\section{Experimental}

\subsection{Sample preparation}

Si NCs were synthesized in a flow-through plasma reactor [33] consisting of a $48 \mathrm{~mm}$ diameter, $610 \mathrm{~mm}$ long glass tube. Both ends were terminated by grounded ultra-torr fittings. The bottom ultra-torr fitting contained a $6 \mathrm{~mm}$ thick stainless steel plate having a small orifice $1 \mathrm{~mm}$ in diameter on the plasma side and opening to $4 \mathrm{~mm}$ diameter on the exit side. The orifice acts as a convergent-divergent aerodynamic nozzle accelerating the effluent gas and creating an expanding particle beam to which the substrate is exposed. $130 \mathrm{~W}, 13.56 \mathrm{MHz} \mathrm{RF}$ power is applied to a $6 \mathrm{~mm}$ tall copper ring electrode positioned $152 \mathrm{~mm}$ above the orifice, 
generating a rotating capacitive filamentary discharge between the ring and lower electrodes and a weak, uniform capacitive glow discharge between the ring and upper electrode. A shutter positioned $50 \mathrm{~mm}$ downstream of the orifice controls exposure of the substrate to the particles. The dislocation-free cubic Si NCs were $20 \mathrm{~nm}$ to $65 \mathrm{~nm}$ in size and had crystallographic facets with slight asperity (see Appendix Figure A2) [34,35].

Sapphire was selected as a substrate in order to minimize compliance effects during compression. The large elastic modulus of sapphire $(340 \mathrm{GPa})$ relative to silicon $(160 \mathrm{GPa})$ results in most of the deformation occurring in the Si NCs. c-axis sapphire squares, $10 \mathrm{~mm}$ by $10 \mathrm{~mm}$ by $0.1 \mathrm{~mm}$, were cleaned ultrasonically. The squares were then shattered into submillimeter pieces. Pieces with sharp tips from the natural cleavage were selected under an optical microscope and mounted on the Hysitron PicoIndenter "chair" using Crystal Bond wax. Silver paste was applied to the base and sides of the sapphire to mitigate charging effects in TEM. The samples were placed in a vacuum oven heated to $50^{\circ} \mathrm{C}$ for at least one hour before NCs deposition. Si NCs were deposited across the edge of the sapphire substrate and isolated particles near the sharp tip were selected for compression experiments to minimize shadowing by the substrate in TEM.

\subsection{Instrumentation}

NCs were compressed by a nominally $100 \mathrm{~nm}$ flat diamond tip of a Hysitron PI95 PicoIndenter modified for improved lateral stability (See Appendix A for details). Compressions were performed in displacement control mode at a rate of $0.8 \mathrm{~nm} \mathrm{sec}-1$, corresponding to a strain rate of about $10^{-2} \mathrm{sec}^{-1}$ to $10^{-1} \mathrm{sec}^{-1}$. Zero-loss-filtered images were acquired using the Gatan 692 TV-rate CCD (25 fps) of a Gatan GIF 2002 attached to an FEI Tecnai F30 (S)TEM with TWIN pole piece having $C_{s}=2.0 \mathrm{~mm}$ and operating at $200 \mathrm{keV}$. Specimens transferred to the TEM in less than one hour remained nominally oxide-free while delays allowed a $1 \mathrm{~nm}$ to $2 \mathrm{~nm}$ oxide layer to form. To improve contrast of dislocations nucleated on the $\{111\}$ slip plane, NCs were rotated for view along the [110] or [1 10$]$ 
directions. Displacement and contact width were measured in-situ and fitted assuming a square contact area to determine the true stress and true strain.

\section{Results and Discussion}

\subsection{Mechanical properties of Si nanocubes}

Si nanocubes (NCs), $20 \mathrm{~nm}$ to $65 \mathrm{~nm}$ in size, were compressed along the [001] direction with acquisition of the load-displacement data. Representative stress-strain curves for four different Si NCs are presented in Figure 1. Contact surfaces areas estimated from simultaneously recorded movies were used to evaluate these true stresses and strains (See Appendix B for details). Five distinct deformation regimes (i-v) are observed in these compression experiments. In regime (i), a linear elastic response was observed upon loading up to the linear elastic (LE) limit at a true strain of $\varepsilon_{\mathrm{T}}^{\mathrm{LE}}=0.07 \pm 0.01$ with a corresponding true stress of $\sigma_{\mathrm{T}}^{\mathrm{LE}}=7.7 \pm 1.1 \mathrm{GPa}$ and resulting elastic modulus $E=110 \pm 22 \mathrm{GPa}$. In regime (ii), stress continued to increase while slightly deviating from linear elastic behavior until reaching the upper yield (UY) point at $\varepsilon_{\mathrm{T}}^{\mathrm{UY}}=0.21 \pm 0.03$, and $\sigma_{\mathrm{T}}^{\mathrm{UY}}=11.6 \pm 1.2 \mathrm{GPa}$. In regime (iii), the upper yield strength was followed by a stress drop, ending at a lower yield (LY) point at $\varepsilon_{\mathrm{T}}^{\mathrm{LY}}=$ $0.35 \pm 0.04$ and $\sigma_{\mathrm{T}}^{\mathrm{LY}}=9.5 \pm 1.2 \mathrm{GPa}$. Stress remained relatively constant in regime (iv) up to $\varepsilon_{\mathrm{T}}=0.45 \pm 0.06$ after which linear work hardening was observed in regime $(\mathrm{v})\left(0.45<\varepsilon_{\mathrm{T}}<\right.$ 0.60). Upon further compression, above $\varepsilon_{\mathrm{T}}>0.60$, work hardening changes slightly and often can be described by a power law (see Appendix $\mathrm{C}$ for additional data).

Analysis of results from 20 individual Si nanocube compression experiments is shown in Figure 2. As can be seen from the Figure 2, the data from oxide-free nanocubes and those with native oxide show no identifiable differences. The linear elastic limit $\left(\varepsilon_{\mathrm{T}}^{\mathrm{LE}}=0.07 \pm 0.01\right)$ in our sub-100 nm Si NCs is about 50\% larger than that observed in crystalline Si nanowires with sub-100 $\mathrm{nm}$ diameter measured in tension [14,15] or by bending [22-26]. However, the 
upper yield point $\sigma_{\mathrm{T}}^{\mathrm{UY}}=11.6 \mathrm{GPa}$ observed here for nanocubes is comparable and approaches the ideal strength of silicon, $E / 10=16 \mathrm{GPa}$ [36]. Interestingly, it appears that for these $\mathrm{Si}$ nanocubes, only weak size dependence might exist for $\varepsilon_{\mathrm{T}}^{\mathrm{LE}}$ and $\sigma_{\mathrm{T}}^{\mathrm{LE}}$ as well as for $\varepsilon_{\mathrm{T}}^{\mathrm{UY}}$ and $\sigma_{\mathrm{T}}^{\mathrm{UY}}$. These values appear to increase with decreasing nanocube size down to a size of $32 \mathrm{~nm}$, and then increase with further decreasing nanocube size. However, the size dependence is not strong and requires further measurements.

A correlation of each region of the stress-strain curve with representative in-situ TEM images illustrates the described deformation regimes. Figure 3 shows all five deformation regimes of a [110] oriented $39 \mathrm{~nm}$ Si NC compression (for details see Supplementary Movie 1). In regime (i), following initial contact, broad strain contours emanate from the contact surfaces and grow across the $\mathrm{NC}$ as strain increases. In this projection the contours are bent, highlighting the asperity of all the NC faces, which includes the $\{001\}$ faces, the $\{111\}$ corner truncations and the $\{110\}$ edge truncations (Figure $3 \mathrm{~g}$ ). When viewed along the [100] projection (Supplementary Movie 2) the strain contours appear to be parallel to the contact surface.

As compression enters regime (ii) and stress-strain curve deviates from linear-elastic response, irregular contrast is observed near the center of contact on the NC. This irregular contrast suggests the creation of a dislocation embryo during compression in this regime. The first activated slip plane occurs in regime (iii) as bands of contrast along $\{111\}$ planes (Figure 3h,i). They form within one to two TEM image frames $(0.04-0.08 \mathrm{sec})$ as the stress reaches its maxima, followed by an observed decrease in stress. Continued compression up to $\varepsilon_{\mathrm{T}}=$ 0.45 , which is now regime (iv), results in the development of additional $\{111\}$ bands of contrast. Within this range, the bands of contrast flicker without change in position and significant multiplication, suggesting that plasticity is accommodated through repeated dislocation activity on the same planes. Finally, as the stress increases into regime (v), 
interlocking $\{111\}$ bands of contrast form, indicating that the interactions between dislocations result in work hardening. Since image contrast in this regime is complex, no reliable post-mortem information about the different activated slip planes can be extracted.

\subsection{Investigation of the mechanisms of plasticity}

Efforts to identify dislocation character in the deformation regimes (ii-iii) described above were also carried out. Having established that dislocation nucleation is occurring on $\{111\}$ planes, nucleation of perfect dislocations with $b=\mathrm{a} / 2<\overline{1} 10>$ and dissociated partial dislocations with $b=\mathrm{a} / 6<11 \overline{2}>$ are both possible. For $[00 \overline{1}]$ compression in $\mathrm{Si}$, the Schmid factor [37] for a $\{111\}<1 \overline{1} 0>$ slip system is 0.408 for all perfect dislocations not perpendicular to the compression axis, while for $\{111\}<11 \overline{2}>$ partial dislocations the two possible Schmid factors are 0.471 and 0.236 (Appendix Figure A2). This suggests that nucleation of partial dislocations is favored in [00 $\overline{1}]$ compression since two of the possible $\{111\}$ slip planes have a higher resolved shear stress compare to a perfect dislocation. The Schmid factor analysis could be further refined for predicting dislocation structures, twinning and phase transformation as in [38].

As discussed earlier, in regime (ii) we observed incipient plasticity revealed by the formation of a small dislocation embryo near the center of the contact surface (see Figure 4ad, and Supplementary Movie 3). This embryo is highlighted by Moiré fringes, which terminate approximately $5 \mathrm{~nm}$ from the center of the contact surface. These fringes suggest that the embryo is a local stress-induced phase transformation. The dislocations appear to be nucleated at the UY point along the interface between the embryo and the host crystal. It should be noted that while predicted in atomistic simulations [28,39-41], this is, to the best of our knowledge, the first reported experimental observation of embryo formation and subsequent dislocation emission from it. It is possible that the embryo could be of $\beta$-tin phase 
Si. Such phase transformations can be induced by hydrostatic stresses as has been observed before in Si under compression [42-47]. Strong friction stresses at the contact surface generate hydrostatic stress at the center of the contact, allowing transformation to the $\beta$-tin phase [27]. Wilkins et al. [47] have reported $\beta$-tin phase transformation in Si under uniaxial compression at around $8 \mathrm{GPa}$ hydrostatic pressure, which is comparable to embryo forming stresses observed here.

To investigate the character of initial dislocations formed in regime (iii), additional targeted experiments were performed. Several Si NCs were compressed where the loading was terminated just after the reaching upper yield point $\left(\varepsilon_{\mathrm{T}}^{\mathrm{UY}}\right)$. High-resolution and dark-field TEM imaging were used to characterize the dislocations inside deformed NCs post-mortem. The results for $41 \mathrm{~nm} \mathrm{Si} \mathrm{NC}$ is shown in Figure 5a. Bright-and-dark contrast on (111) planes can be seen after compression in the bright-field (BF) image (Figure 5b). The selected area diffraction pattern obtained after compression (Figure 5c) shows two defect-related features:

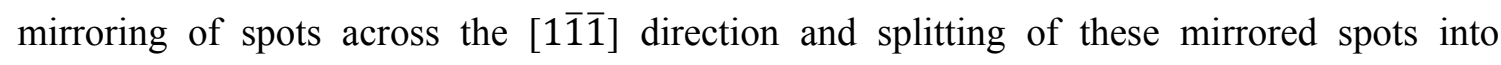
multiple low intensity spots. The mirroring indicates the presence of faulted $(\overline{1} \overline{1} \overline{1})_{\mathrm{f}}$ planes, due to the overlap of misaligned crystal regions, while the splitting of spots indicates of presence of a thin faulted region. This is confirmed by post-mortem by dark-field (DF) TEM imaging with the $(\overline{1} \overline{1} \overline{1})$ and the faulted $(\overline{1} \overline{1} \overline{1})$ f reflections (Figure $5 \mathrm{~d}, \mathrm{e})$. As can be seen, the $(\overline{1} \overline{1} \overline{1})$ f reflections correspond to a thin $5 \mathrm{~nm}$ region of the NC. Such faults must be formed by nucleation of a leading partial dislocation.

Differentiation between isolated stacking faults or twin bands was not possible from these results, since the spatial resolution in DF-TEM images about faulted planes was limited to a few nanometers, similar to the width of potential microtwins [48]. In-situ DF-TEM images obtained using the $(2 \overline{2} 0)$ reflection from a NC viewed along its [110] zone axis (Supplementary Figure S3 and Supplementary Movie 4) revealed Moiré fringes normal to the 
predicted leading partial dislocation with $b=\mathrm{a} / 6[\overline{1} \overline{1} 2]$ or $b=\mathrm{a} / 6[112]$. These fringes (Figure 5f) can be explained by crystalline mismatch created by leading partial dislocations on the inclined $\{111\}$ planes.

Observation of initial $\{111\}$ contrast in high-resolution TEM image of compressed NCs is also consistent with nucleation of partial dislocations. As can be seen from Figure 6a, two bands of contrast extending from the center of the top and bottom contact surfaces towards opposing corners of the NC, respectively. Fourier filtering of the micrograph (keeping only the (111) reflection), as shown in Figure 6b, reveals that the outer portions of the crystal are faulted with respect to the center, which is indicative of stacking faults separating the three regions of the crystal. Such stacking faults must be formed by nucleation of a leading partial dislocation. We interpret that the dislocations are nucleated at the center of the contact and terminate at an opposing truncated corner of the nanocube. The opposite may occur since stress concentrations at the corner would result in easier nucleation as seen in pillars [49], but this should result in an earlier onset of hardening than observed here

Additional compression towards the end of regime (iii) leads to the activation of numerous parallel $\{111\}$ slip planes (Figure 6c,d). No microtwins can be seen here suggesting that nucleation of independent stacking faults is favored over nucleation on adjacent slip planes, i.e. deformation twinning. Furthermore, it was observed that the $\{111\}$ contrast bands do not appear to multiply considerably prior to hardening, suggesting that the same slip planes are repeatedly slipping. Because the contrast bands also do not disappear, the trailing partials must quickly nucleate and pass through the $\mathrm{NC}$ on the same slip plane to allow for subsequent leading-trailing partial dislocation pairs.

Having identified $\{111\}\langle 11 \overline{2}\rangle$ partial dislocations without substantial twinning as the mechanism of plasticity in these NCs, it can be argued that the plasticity mechanism in Si crystals changes at some characteristic length-scale. Conventionally, at high-stress (above 1 
$\mathrm{GPa}$ ) and low temperature (below $400^{\circ} \mathrm{C}$ ), the plasticity in bulk $\mathrm{Si}$ is accommodated by the nucleation of perfect dislocations, particularly under large confining pressures $[36,50]$. This was also confirmed down to $\mu \mathrm{m}$-scale by Korte and Clegg [51] using $2 \mu \mathrm{m}$ Si pillars at $100{ }^{\circ} \mathrm{C}$. These results suggest that a transition from perfect to partial dislocation as the dominant plasticity mechanism occurs at a length scale between micrometer to sub-100 nm. At larger scales, the statistical likelihood of defects also increases. If dislocation nucleation was not determined by the theoretical strength anymore, as described by Li et al. [52], this could affect the dominant plasticity mechanism.

High resolution imaging of the dislocation core could provide much more definitive answers regarding why partial dislocations are favored at the sub-100 $\mathrm{nm}$ scale in compression as observed here. Attempts were made to understand the defect core structure by HR-TEM, however definitive interpretation was not possible due to the non-planar surfaces of the projected NCs, the varying location of the dislocation, and experimental limits of specimen tilting. The lack of a unique solution is apparent when TEM exit wave images for a stacking fault and screw dislocation in these NCs are compared (see Appendix D for details).

\section{Conclusion}

Silicon NCs, $20 \mathrm{~nm}$ to $65 \mathrm{~nm}$ in size range and having $\{100\}$ facets with slight edge/corner truncation were compressed along the [001] direction. The linear elastic strain limit was found to be $\varepsilon \mathrm{T}=0.07$. Upper yield stresses of $11 \mathrm{GPa}$ at $\varepsilon \mathrm{T}=0.20$ were observed before plasticity occurs by activation of $\{111\}<11 \overline{2}>$ slip systems. A practical size invariance of upper and lower yield stresses, corresponding strains, and elastic modulus were observed over the range tested, suggesting that the reduction of size no longer a factor for dislocation nucleation and propagation. Dislocation embryos, which appear to be $\beta$-tin phase $\mathrm{Si}$, were observed to form and initiate plasticity. The hydrostatic stresses develop at the contact surface due to friction can be responsible for producing these $\beta$-tin phase embryos. Substantial plastic 
deformation by partial dislocations with $b=\mathrm{a} / 6<11 \overline{2}>$ slipping in $\{111\}$ planes was also observed, in agreement with calculated Schmid factors. 


\section{Acknowledgements}

This work was supported in part by NSF MRSEC under awards DMR-0819885 and DMR-1420013. STEM analysis was carried out in the Characterization Facility of the University of Minnesota, which receives partial support from the NSF through the MRSEC program. Multislice computer simulations were performed using resources provided by the Minnesota Supercomputing Institute. The authors would like to thank Doug Stauffer and Ryan Major of Hysitron for their continued support.

\section{Appendix A: Indenter Stabilization}

For the in-situ TEM Hysitron PicoIndenter system, a significant portion of the indenter shaft is suspended in vacuum beyond the final point of stabilization and is sensitive to acoustic interference. The vibrations can strongly affect the mechanical data and TEM imaging of particle compression. Such vibrations in contact with a $30 \mathrm{~nm}$ crystal amount to about 0.1 transverse strain for the silicon nanocubes. This strain and the associated stresses have resolved shear stress components contributing to dislocation nucleation which are not measured by the axial load-sensing transducer.

To stabilize the indenter, the shaft was pressed against the wall of the holder near the MEMS transducer, reducing the vibration from $10 \mathrm{~nm}$ amplitude to below $1 \mathrm{~nm}$. The resulting load-displacement data was improved from that obtained by the original vibrating tip, Appendix Figure A1. Compression of a Si NC with a vibrating tip exhibits numerous load drops of $\sim 2-3 \mu \mathrm{N}$ which constitutes a substantial portion of the applied load. With reduced vibration operation force-displacement curves show drastically reduced load drops, such that they were virtually indiscernible while also increasing the resolved applied load with respect to displacement.

After these initial observations, a more refined solution to this issue was introduced using two adjustments. First, the development of a direct-drive coarse adjustment stage by Hysitron reduced the lateral vibrations from roughly $10 \mathrm{~nm}$ amplitude to below $5 \mathrm{~nm}$ while significantly improving the ability to make small coarse adjustments of indenter position. Secondly, to reduce lateral vibrations further, we inserted polyimide foam with large 
elasticity, low memory, and vacuum capability between the indenter shaft and holder wall. The small pressure exerted on the indenter shaft was found to reduce vibration beyond detection while simultaneously allowing coarse adjustment of indenter position.

\section{Appendix B: Calculation of True Stress \& True Strain}

An additional visual aid for the particle shape, slip planes and possible Burger's vectors is shown in Appendix Figure A2. As can be observed, the primary facets are $\{100\}$, with $\{111\}$ corner truncations and $\{110\}$ edge truncations. The truncation of edges and corners leads to the rounded profile of $<110>$-oriented NCs.

Analysis is simplified by direct-observation of the contact surfaces of NCs compressed on flat $\{100\}$ cube facets between a diamond tip and sapphire substrate, both materials with substantially higher modulus than Si. Stresses in the center of the particle are much lower than at the contact surfaces, making observation of contact surfaces necessary for accurate stress determination. The stress gradient from the contact to the center of the NCs results from both a slight natural truncation as well as from barreling as compression proceeds due to contact friction. For most compressions, instantaneous particle height and contact width, wi, were visible throughout the in-situ testing, allowing the instantaneous contact stress to be calculated as $\sigma_{i}=L_{i} / w_{i}^{2}$, where $L_{i}$ is the instantaneous load.

In the event that the contact surface was obstructed a displacement-dependent exponential fitting function to model the asperity of the $\mathrm{NC}$ surface was employed to determine contact stress. Under assumption of $\mathrm{NC}$ volume conservation the effective contact width was approximated as:

$$
w_{i}=w_{0}+\left(d_{0}-w_{0}\right)\left[1-\exp \left(-6 \varepsilon_{i}\right)\left(1-\varepsilon_{i}\right)^{-v / 2}\right]
$$

where $w_{0}$ is the initial contact width, $d_{0}$ is the initial $\mathrm{NC}$ width, $\varepsilon_{i}$ is instantaneous axial strain, and $v$ is the Poisson ratio. The surface asperity and corrects the contact area at low strains are taken into account here as well as volumetric expansion at large strains. The model 
reproduces the cases where contact surfaces were visible, as demonstrated in Appendix Figure A3.

\section{Appendix C: Additional Experimental Data}

A complete data set of true-stress true-strain curves is presented in Appendix Figure A4. In part a, complete tests are shown, while in part b, tests that were terminated after the upper yield point are shown (this was done for the purposes of identifying initial dislocation character, as described in Section 3.2).

An additional composite of TEM images and load-displacement curve is shown for a nanocube compressed under in-situ dark field conditions in Appendix Figure A5. Calculation of true stress and true strain was challenging due to the decreased visibility of the contact width in dark field, particularly at higher strains. The red-marked Moire pattern displayed in the fourth frame provides additional evidence of slip by partial dislocations, as shown and discussed in Figure $5 \mathrm{f}$ and Section 3.2, respectively.

\section{Appendix D: Multislice Simulations}

Multislice simulations [53] of TEM exit waves for perfect screw dislocation and stacking fault in a $37 \mathrm{~nm} \mathrm{NC}$ are shown respectively in Appendix Figure A6 and Appendix Figure A7. Simulation parameters were: $V=200 \mathrm{keV}, C_{s}=2.0 \mathrm{~mm}$, pixel resolution was 0.15 $\AA$ pixel $^{-1}$ on a $4 \mathrm{k} \mathrm{x} 4 \mathrm{k}$ pixel $^{2}$ grid. Introduction of $0^{\circ}$ to $4^{\circ}$ rotation of the $\mathrm{NC}$ about the $[1 \overline{1} \overline{1}]$

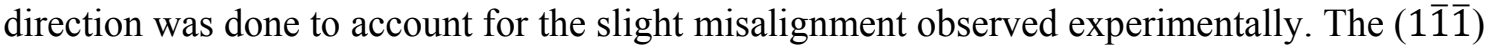
- Fourier filtered images of the Multislice exit waves are shown in Appendix Figure A8 for a NC containing a single screw dislocation and another NC containing a single stacking fault. Results at $0^{\circ}, 1^{\circ}, 2^{\circ}$ and $4^{\circ}$ tilt show different set of planar fringes, which can be misinterpreted as either partial screw dislocations or partial edge dislocations or $60^{\circ}$ dislocations or stacking faults. This ambiguity in observing dislocations by HR-TEM given 
by the nanocube morphology and experimental constraints should be considered before making any conclusion about the dislocation character.

\section{References}


[1] J. Li, Z. Shan, E. Ma, "Elastic strain engineering for unprecedented materials properties, MRS Bull. 39 (2014) 108-114.

[2] D. G. Schlom, L.-Q. Chen, C. J. Fennie, V. Gopalan, D. A. Muller, X. Pan, R. Ramesh, R. Uecker, Elastic strain engineering of ferroic oxides, MRS Bull. 39 (2014) 118130.

[3] M. J. Hÿtch, A. M. Minor, Observing and measuring strain in nanostructures and devices with transmission electron microscopy, MRS Bull. 39 (2014) 138-146.

[4] B. Yildiz, "Stretching" the energy landscape of oxides-Effects on electrocatalysis and diffusion, MRS Bull. 39 (2014) 147-156.

[5] D. Yu, J. Feng, J. Hone, Elastically strained nanowires and atomic sheets, MRS Bull.39 (2014) 157-162.

[6] T. Zhu, J. Li, Ultra-strength materials, Prog. Mater. Sci.55 (2010) 710-757.

[27] S. W. Bedell, A. Khakifirooz, D. K. Sadana, Strain scaling for CMOS, MRS Bull. 39 (2014) 131-137.

[38] M. D. Uchic, D. M. Dimiduk, J. N. Florando, W. D. Nix, Sample dimensions influence strength and crystal plasticity, Science 305 (2004) 986-989.

[9] J. R. Greer, W. C. Oliver, W. D. Nix, Size dependence of mechanical properties of gold at the micron scale in the absence of strain gradients, Acta Mater. 53 (2005) 1821-1830.

[10] M. R. Sørensen, M. Brandbyge, K. W. Jacobsen, Mechanical deformation of atomicscale metallic contacts: structure and mechanisms, Phys. Rev. B:Condens. Matter Mater. Phys. 57 (1998) 3283.

[11] F. Östlund, K. Rzepiejewska-Malyska, K. Leifer, L. M. Hale, Y. Tang, R. Ballarini, W. W. Gerberich, J. Michler, Brittle-to-Ductile Transition in Uniaxial Compression of Silicon Pillars at Room Temperature, Adv. Func. Mater. 19 (2009) 2439-2444. 
[12] Y. Zhu, F. Xu, Q. Qin, W. Y. Fung, W. Lu, Mechanical properties of vapor- liquidsolid synthesized silicon nanowires, Nano Lett. 9 (2009) 3934-3939.

[13] T. Tsuchiya, Tensile testing of silicon thin films, Fatigue Fract. Eng. Mater. Struct. 28 (2005) 665-674.

[14] X. D. Han, K. Zheng, Y. F. Zhang, X. N. Zhang, Z. Zhang, Z. L. Wang, Lowtemperature in situ large-strain plasticity of silicon nanowires, Adv. Mater. 19 (2007) 2112.

[15] R. A. Minamisawa, M. J. Süess, R. Spolenak, J. Faist, C. David, J. Gobrecht, K. K. Bourdelle, H. Sigg, Top-down fabricated silicon nanowires under tensile elastic strain up to 4.5\%, Nat. Commun. 3 (2012) 1096.

[16] W. W. Gerberich, W. M. Mook, C. R. Perrey, C. B. Carter, M. I. Baskes, R. Mukherjee, A. Gidwani, J. Heberlein, P. H. McMurry, S. L. Girshick, Superhard silicon nanospheres, J. Mech. Phys. Solids 51 (2003) 979-992.

[17] T. Namazu, Y. Isono, T. Tanaka, Evaluation of size effect on mechanical properties of single crystal silicon by nanoscale bending test using AFM, J. Microelectromech. Syst 9 (2000) 450-459.

[18] Y. Sun, S. E. Thompson, T. Nishida, Physics of strain effects in semiconductors and metal-oxide-semiconductor field-effect transistors, J. Appl. Phys. 101 (2007) 104503.

[19] P. Hashemi, L. Gomez, J. L. Hoyt, Gate-all-around n-MOSFETs with uniaxial tensile strain-induced performance enhancement scalable to sub-10-nm nanowire diameter, IEEE Electron Device Lett. 30 (2009) 401-403.

[20] V. Pott, K. E. Moselund, K. E. Moselund, D. Bouvet, L. De Michielis, A. M. Ionescu, Nanotechnology, IEEE Trans. Nanotechnol. 7 (2008) 733.

[21] M. Chu, Y. Sun, U. Aghoram, S. E. Thompson, Strain: a solution for higher carrier mobility in nanoscale MOSFETs, Ann. Rev. Mater. Res. 39 (2009) 203-229. 
[22] K. Zheng, X. Han, L. Wang, Y. Zhang, Y. Yue, Y. Qin, X. Zhang, Z. Zhang, Atomic mechanisms governing the elastic limit and the incipient plasticity of bending Si nanowires, Nano Lett. 9 (2009) 2471-2476.

[23] L. Wang, K. Zheng, Z. Zhang, X. Han, Direct atomic-scale imaging about the mechanisms of ultralarge bent straining in Si nanowires, Nano Lett. 11 (2011) 2382-2385.

[24] S. Hoffmann, I. Utke, B. Moser, J. Michler, S. H. Christiansen, V. Schmidt, S. Senz, P. Werner, U. Gösele, C. Ballif, Measurement of the bending strength of vapor-liquid-solid grown silicon nanowires, Nano Lett. 6 (2006) 622-625.

[25] D.-M. Tang, C.-L. Ren, M.-S. Wang, X. Wei, N. Kawamoto, C. Liu, Y. Bando, M. Mitome, N. Fukata, D. Golberg, Mechanical properties of Si nanowires as revealed by in situ transmission electron microscopy and molecular dynamics simulations, Nano Lett. 12 (2012) 1898-1904.

[26] G. Stan, S. Krylyuk, A. V. Davydov, I. Levin, R. F. Cook, Ultimate bending strength of si nanowires, Nano Lett. 12 (2012) 2599-2604.

[27] D. Chrobak, N. Tymiak, A. Beaber, O. Ugurlu, W. W. Gerberich, R. Nowak, Deconfinement leads to changes in the nanoscale plasticity of silicon, Nat. Nanotechnol. 6 (2011) 480-484.

[28] N. Zhang, Q. Deng, Y. Hong, L. Xiong, S. Li, M. Strasberg, W. Yin, Y. Zou, C. R. Taylor, G. Sawyer, Y. Chen, Deformation mechanisms in silicon nanoparticles, J. Appl. Phys. $109(2011) 063534$.

[29] J. Guénolé, S. Brochard, J. Godet, Unexpected slip mechanism induced by the reduced dimensions in silicon nanostructures: Atomistic study, Acta Mater. 59 (2011) 7464-7472.

[30] J. Godet, L. Pizzagalli, S. Brochard, P. Beauchamp, Computer study of microtwins forming from surface steps of silicon, Comput. Mater. Sci. 30 (2004) 16-20.

[31] P. Valentini, W. W. Gerberich, T. Dumitrică, Phase-transition plasticity response in uniaxially compressed silicon nanospheres, Phys. Rev. Lett. 99 (2007) 175701. 
[32] L. Pizzagalli, J. Godet, J. Guénolé, S. Brochard, E. Holmstrom, K. Nordlund, T. Albaret, A new parametrization of the Stillinger-Weber potential for an improved description of defects and plasticity of silicon, J. Phys.: Condens. Matter 25 (2013) 055801.

[33] A. Bapat, M. Gatti, Y. P. Ding, S. A. Campbell, U. Kortshagen, A plasma process for the synthesis of cubic-shaped silicon nanocrystals for nanoelectronic devices, J. Phys. D: Appl. Phys. 40 (2007) 2247.

[34] A. Bapat, C. Anderson, C. R. Perrey, C. B. Carter, S. A. Campbell, U. Kortshagen, Plasma synthesis of single-crystal silicon nanoparticles for novel electronic device applications, Plasma Phys. Controlled Fusion 46 (2004) B97.

[35] A. J. Wagner, C. M. Anderson, J. N. Trask, L. Cui, A. Chov, K. A. Mkhoyan, U. R. Kortshagen, Propagating Nanocavity-Enhanced Rapid Crystallization of Silicon Thin Films Nano Lett. 13 (2013) 5735-5739.

[36] J. Frenkel, Zur theorie der elastizitätsgrenze und der festigkeit kristallinischer körper, Z. Physik 37 (1926) 572-609.

[37] E. Schmid, W. Boas, F. I. G. Rawlins, Kristallplastizität, The Journal of Physical Chemistry 39 (1935) 1248.

[38] J. Kwon, M. C. Brandes, P. S. Phani, A. P. Pilchak, Y. F. Gao, E. P. George, G. M. Pharr, M. J. Mills, Characterization of deformation anisotropies in an $\alpha$-Ti alloy by nanoindentation and electron microscopy, Acta Mater. 61 (2013) 4743-4756.

[39] J. Li, K. J. Van Vliet, T. Zhu, S. Yip, S. Suresh, Atomistic mechanisms governing elastic limit and incipient plasticity in crystals, Nature 418 (2002) 307-310.

[40] K. J. Van Vliet, J. Li, T. Zhu, S. Yip, S. Suresh, Quantifying the early stages of plasticity through nanoscale experiments and simulations, Phys. Rev. B 67 (2003) 104105.

[41] I. Salehinia, S. K. Lawrence, D. F. Bahr, The effect of crystal orientation on the stochastic behavior of dislocation nucleation and multiplication during nanoindentation, Acta Mater. 61 (2013) 1421-1431. 
[42] D. L. Callahan, J. C. Morris, The extent of phase transformation in silicon hardness indentations, J. Mater. Res. 7 (1992) 1614.

[43] I. V. Gridneva, Y. V. Milman, V. I. Trefilov, Phase transition in diamond-structure crystals during hardness measurements, Phys. Status Solidi (a) 14 (1972) 177-182.

[44] G. M. Pharr, W. C. Oliver, D. R. Clarke, The mechanical behavior of silicon during small-scale indentation, J. Electron. Mater. 19 (1990) 881-887.

[45] V. Domnich, Y. Gogotsi, S. Dub, Effect of phase transformations on the shape of the unloading curve in the nanoindentation of silicon, Appl. Phys. Lett. 76 (2000) 2214-2216.

[46] G. M. Pharr, W. C. Oliver, D. S. Harding, New evidence for a pressure-induced phase transformation during the indentation of silicon, J. Mater. Res. 6 (1991) 1129-1130.

[47] R. G. Hennig, A. Wadehra, K. P. Driver, W. D. Parker, C. J. Umrigar, J. W. Wilkins, Phase transformation in Si from semiconducting diamond to metallic $\beta$-Sn phase in QMC and DFT under hydrostatic and anisotropic stress, Phys. Rev. B 82 (2010) 014101.

[48] D. Williams, C. Carter, Transmission electron microscopy: a textbook for materials science, Springer Verlag, 2009.

[49] H. Bei, Y. F. Gao, S. Shim, E. P. George, and G. M. Pharr, Strength differences arising from homogeneous versus heterogeneous dislocation nucleation, Phys. Rev. B 77 (2008) 060103.

[50] J. Rabier, L. Pizzagalli, J. Demenet, Dislocations in silicon at high stress, Dislocat. Solids 16 (2010) 47-108.

[51] S. Korte, J. S. Barnard, R. J. Stearn, W. J. Clegg, Deformation of silicon-insights from microcompression testing at 25-500 C, Int. J. Plast. 27 (2011) 1853-1866.

[52] T. L. Li, H. Bei, J. R. Morris, E. P. George, Y. F. Gao, Scale effects in convoluted thermal/spatial statistics of plasticity initiation in small stressed volumes during nanoindentation, Mat. Sci. Tech. Ser. 28 (2012) 1055-1059.

[53] E. J. Kirkland, Advanced computing in electron microscopy, Springer, 2010. 
Figure 1. (a) HR-TEM image of a $39 \mathrm{~nm}$ [1110]-oriented Si NC on sapphire substrate. Inset shows high-resolution fringes corresponding to [110] orientation of the Si crystal. Scale bar is $10 \mathrm{~nm}$. (b) Schematic illustration of the NC shown in (a), with top and bottom surface representing indenter and substrate. Arrows indicate direction of load application on the NC. (c) Representative true stress vs. true strain plots for NCs of varying sizes showing five distinct deformation regimes.

Figure 2. (a) True strain at LE limit and UY point as a function of NC size. Large LE strains (black squares) of about $7 \%\left(\varepsilon_{\mathrm{t}}^{\mathrm{LE}}=0.07\right)$ with strains at the UY point (red circles) approaching $20 \%\left(\varepsilon_{\mathrm{T}}^{\mathrm{UY}}=0.20\right)$ are observed. (b) Yield strength at LE limit, $\sigma_{\mathrm{T}}^{\mathrm{LE}}$ (black squares) and at the UY point, $\sigma_{\mathrm{T}}^{\mathrm{UYP}}$ (red circles) as a function of NC size. The $\sigma_{\mathrm{T}}^{\mathrm{LE}}$ approaches $8 \mathrm{GPa}$ and $\sigma_{\mathrm{T}}^{\mathrm{UY}}$ exceeds $11 \mathrm{GPa}$. Solid symbols are for nominally oxide-free nanocubes while open symbols are for NCs with native oxide. Fitted dotted lines represent possible trend reversal at $32 \mathrm{~nm} \mathrm{NC}$ size.

Figure 3. (a-e) In-situ TEM image series with its corresponding true stress vs true strain curve (f) for a $39 \mathrm{~nm}[\overline{110}]$-oriented Si NC. (a) The first point of contact of the indenter with the NC. (b) At $\varepsilon \mathrm{T}<0.07$, symmetric strain contours are observed to extend from the compressed surfaces. (c) Sharp contrast bands on $\{111\}$ planes appear at the UY point. (d) The bands multiply as strain increases, until (e) complex dislocation activity is observed at $\varepsilon \mathrm{T}>0.39$. Scale bar is $20 \mathrm{~nm}$. (g) A model depicting the morphology of a truncated $39 \mathrm{~nm} \mathrm{Si} \mathrm{NC} \mathrm{with}$ crystallographic planes forming the truncations shown. (h) Inside view of Si NC model shown in $(\mathrm{g})$ with highlighted activated slip planes under [001] compression. (i) Thompson tetrahedron explaining the $[110]$ viewing projection with the $\{111\}$ slip planes forming the faces of the tetrahedra.

Figure 4. In-situ TEM image series of a $45 \mathrm{~nm}$ Si NC with [110] direction oriented along the electron beam direction and slip planes inclined to the beam direction. (a) Initial contact of the indenter with the NC. A model with orientation of the NC is shown. (b) Further compression of $\mathrm{NC}$ leads to development of irregular contrast bands at the indenter-NC interface. (c) A embryo ( $\sim 5 \mathrm{~nm}$ in size) forms at the upper contact surface as the LE limit is reached. The highlighted region shows Moiré fringes at dislocation embryo. (d) At the UY point, $\{111\}$ contrast develops, extending from the embryo to the opposing corner. Parallel contrast bands form on the lower contact surface as well. A model shows activated $\{111\}$ slip planes inside the NC. Scale bar is $20 \mathrm{~nm}$.

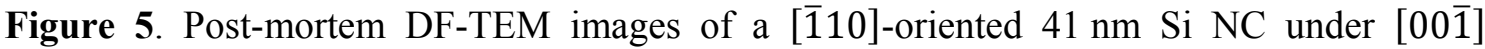
compression. (a),(b) Pre- and post-compression HR-TEM image. (c) Selected area diffraction pattern of the compressed NC shown in (b) aligned such that the indenter contributes minimal diffraction. Circled spots are mirrored about [1 $1 \overline{1} \overline{1}]$ direction indicating faulting on (111) and $(\overline{1} \overline{1} \overline{1})$ planes. (d) Post-mortem DF-TEM image obtained using ( $\overline{1} \overline{1} \overline{1})$ reflection showing bright contrast along the faulted region. (e) DF-TEM image obtained using the ( $(\overline{1} \overline{1} \overline{1}) \mathrm{f}$ spot as shown in (c) by the streaked region. Bright region corresponds to the fault planes. (f) In-situ DF-TEM image obtained using (2$\overline{2} 0)$ spot when the NC is viewed along [110] direction. This shows the same (111) slip plane shown in (d) and (e) from a different direction. The models in yellow represent the NC and its orientation and the activated $\{111\}$ slip planes inside it. Scale bar is $10 \mathrm{~nm}$. 
Figure 6. (a) Post-mortem BF-TEM image and (b) respective (111) Fourier-filtered image of [110]-oriented $36 \mathrm{~nm}$ Si NC compressed to the UY point revealing two stacking faults. (c) Post-mortem BF-TEM image and (d) respective (111) Fourier-filtered images of [110]oriented $28 \mathrm{~nm}$ Si NC compressed to the LY point. Numerous, closely spaced, stacking faults on its (111) planes can be observed. Scale bar is $10 \mathrm{~nm}$.

Appendix Figure A1: Force vs. displacement curves for Si NCs with lateral (out of contact) vibrations (cyan), and after damping vibrations to less than $1 \mathrm{~nm}$ with indenter modifications (green). Load-drops type behavior seen with standard commercial non-vibration-damped indenters could be mistakenly interpreted as pop-in events from dislocation bursts during stable compression rather than unresolved shear stresses contributing to deformation.

Appendix Figure A2: Thompson tetrahedron and schematic of slip planes in the Si NCs. (a) [110] projection of Si NCs. (b) HR-TEM image of a $38 \mathrm{~nm}$ [1110]-oriented Si NC. (c) View along the $[\overline{1} 10]$ direction with the $(\overline{1} \overline{1} 1)$ and (111) active slip planes and (d) the respective Thompson tetrahedron defining slip planes and slip directions with Greek letters indicating each slip plane. (e) View along the [110] direction with the (111) slip plane highlighted and (f) its Thompson tetrahedron. The directions with highest Schmid factors are highlighted by dashed red line in (e) and (f).

Appendix Figure A3: True stress vs true strain curve as determined from instantaneous contact width (red) and using a fitted contact area (blue). The fit matches the experimental curve with only slight deviation in the region of the load drop.

Appendix Figure A4: (a) True stress vs true strain curves for [001]-compressed NCs of varying size. (b) True stress vs true strain curves for [001] -compressed NC of varying size. For post-mortem HR-TEM analysis, the compressions were aborted as soon as dislocation activity was observed.

Appendix Figure A5: (a) In-situ DF-TEM image with $\mathrm{g}=(2 \overline{2} 0)$ of a Si NC under [00 $\overline{1}]$ compression. NC is oriented such that its [110] zone axis is oriented along the beam direction. The full, inclined slip plane, highlighted in red in $17.82 \mathrm{~s}$ frame, can be seen starting across the width of the top surface and extending to a corner of the lower surface of the NC outlined in blue. (b) Corresponding force vs displacement curve.

Appendix Figure A6: (a) Ball and stick model of a single (1 $\overline{1} 1)[10 \overline{1}]$ screw dislocation in a [110]-oriented $37 \mathrm{~nm} \mathrm{Si} \mathrm{NC} \mathrm{and} \mathrm{(b)} \mathrm{the} \mathrm{resulting} \mathrm{amplitude} \mathrm{of} \mathrm{the} \mathrm{TEM} \mathrm{exit} \mathrm{wave} \mathrm{(} V=$ $200 \mathrm{keV}, C_{S}=2.0 \mathrm{~mm}$, pixel resolution of $0.15 \AA$ pixel $^{-1}$ on a $4 \mathrm{k} \mathrm{x} 4 \mathrm{k}$ pixel $^{2}$ grid). (c),(d) Magnified view of the screw dislocation from (a) and (b) respectively.

Appendix Figure A7: (a) Ball and stick model of a single (1 $\overline{1} 1)[1 \overline{1} \overline{2}]$ partial dislocation in a [110]-oriented $37 \mathrm{~nm} \mathrm{Si} \mathrm{NC} \mathrm{and} \mathrm{(b)} \mathrm{the} \mathrm{resulting} \mathrm{amplitude} \mathrm{of} \mathrm{the} \mathrm{TEM} \mathrm{exit} \mathrm{wave}(V=$ $200 \mathrm{keV}, C_{s}=2.0 \mathrm{~mm}$, pixel resolution of $0.15 \AA$ pixel $^{-1}$ on a $4 \mathrm{k} \mathrm{x} 4 \mathrm{k}$ pixel $^{2}$ grid). (c),(d) Magnified view of the partial dislocation from (a) and (b) respectively.

Appendix Figure A8: A comparison of the (11̄1)-Fourier filtered amplitude of the simulated TEM exit wave $\left(V=200 \mathrm{keV}, C_{s}=2.0 \mathrm{~mm}\right.$, pixel resolution of $0.15 \AA$ pixel $^{-1}$ on a $4 \mathrm{k} \mathrm{x} 4 \mathrm{k}$ pixel $^{2}$ grid) for inclined $(1 \overline{1} 1)[10 \overline{1}]$ screw dislocation and $(1 \overline{1} 1)[1 \overline{1} \overline{2}]$ stacking fault in a $37 \mathrm{~nm}$ Si NC. Misalignment of the NC causes the planar fringes to change considerably making interpretation of dislocation character difficult. 
a

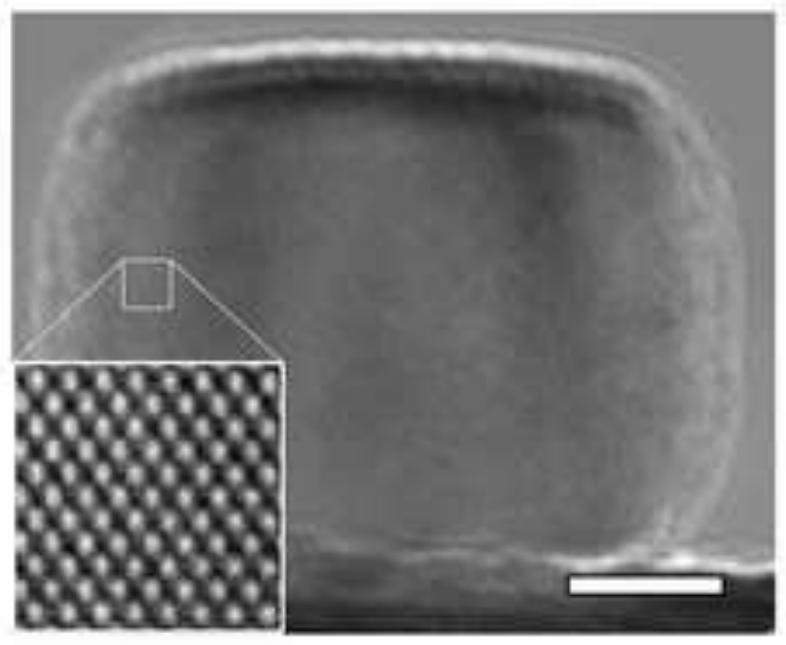

b

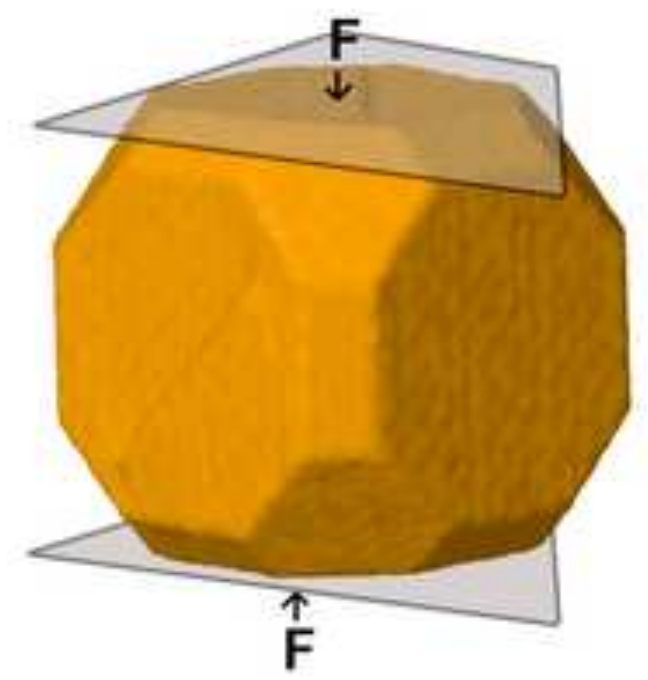

C

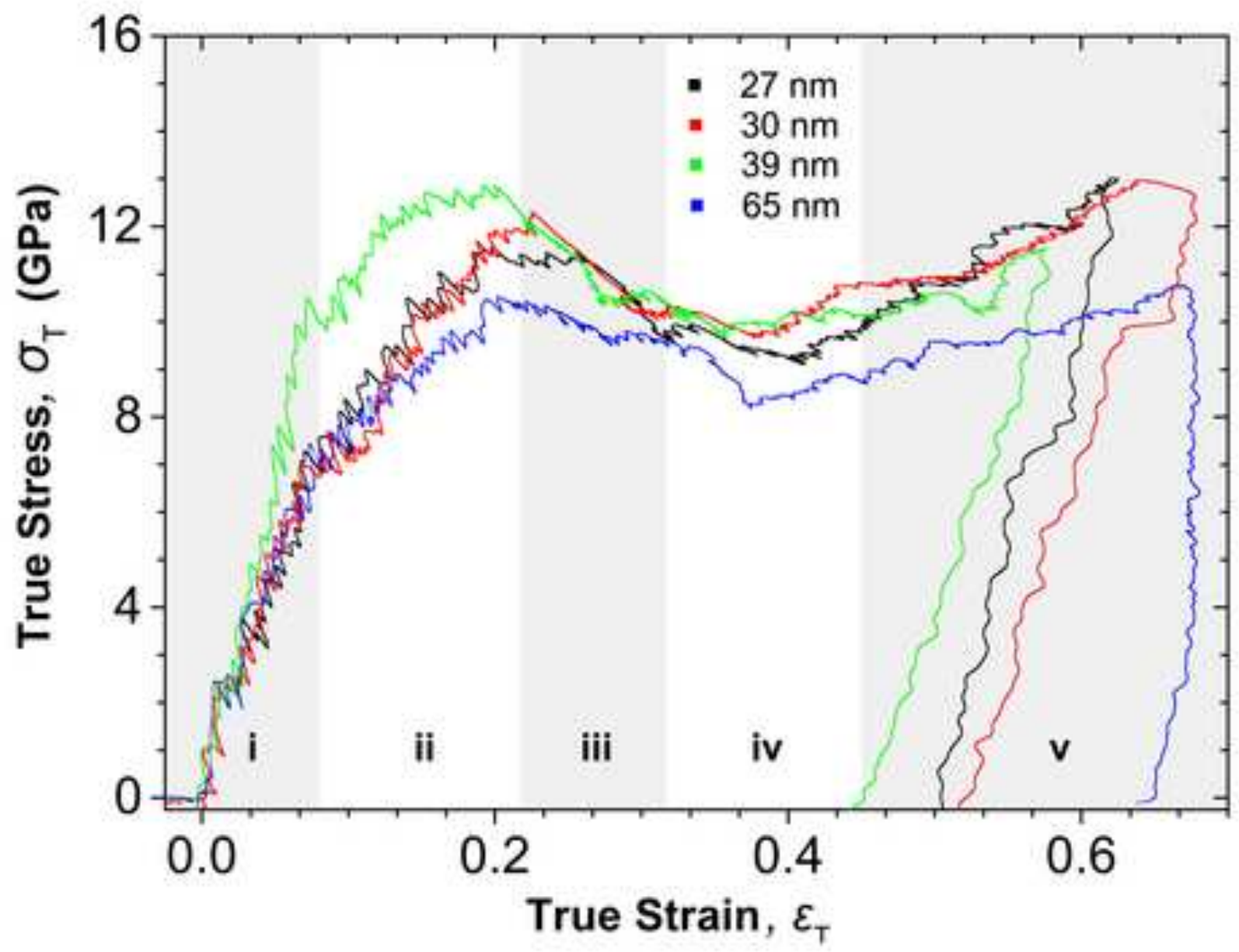



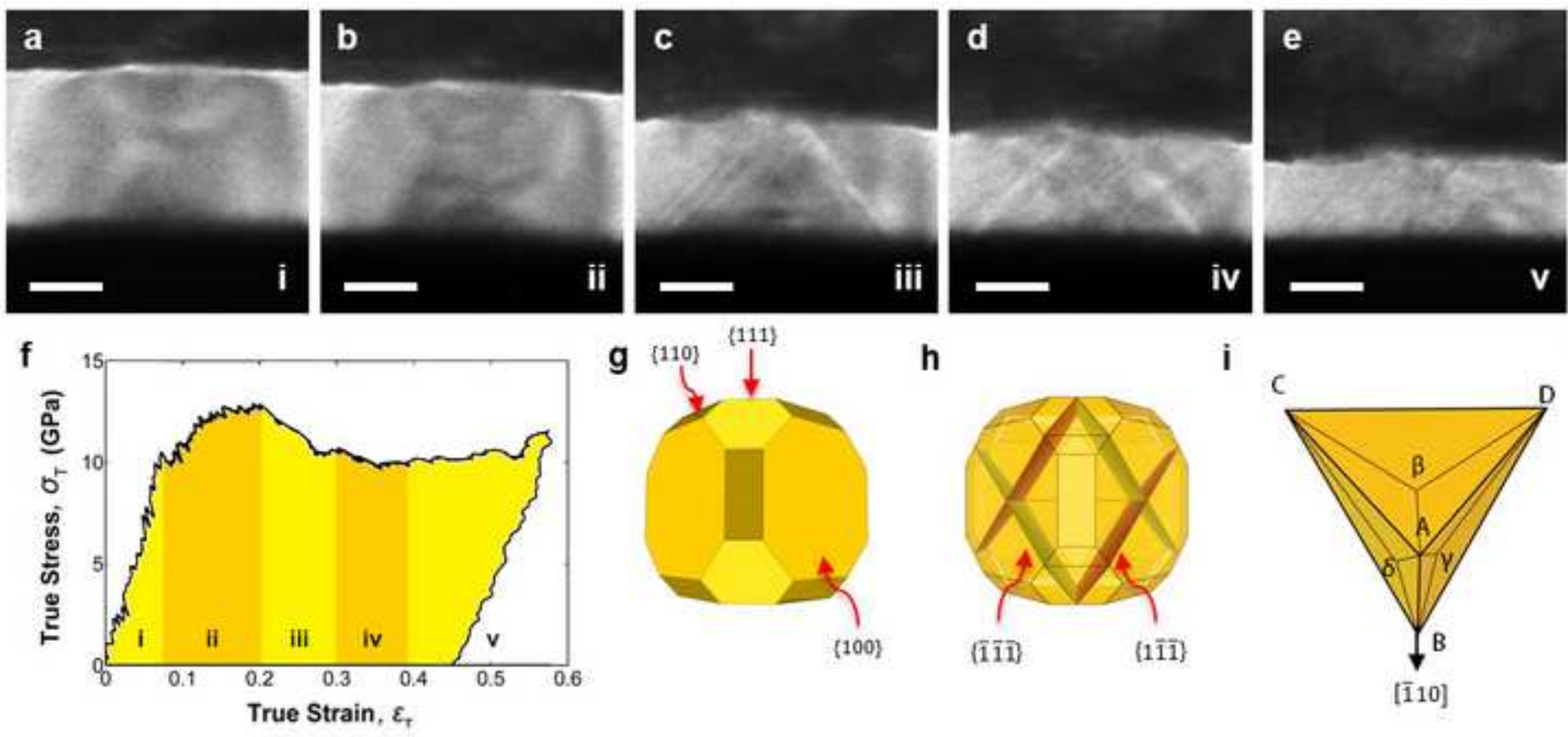

h

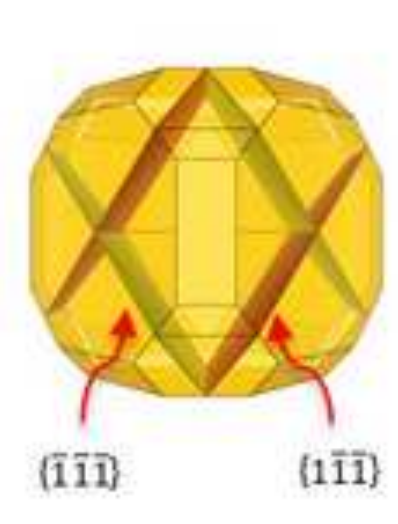
i

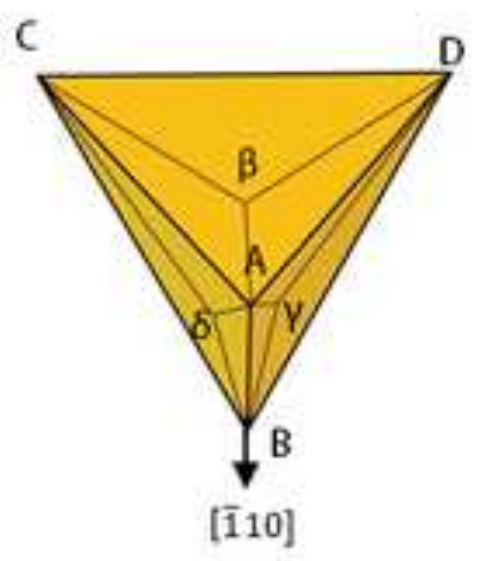

00)

[110] 

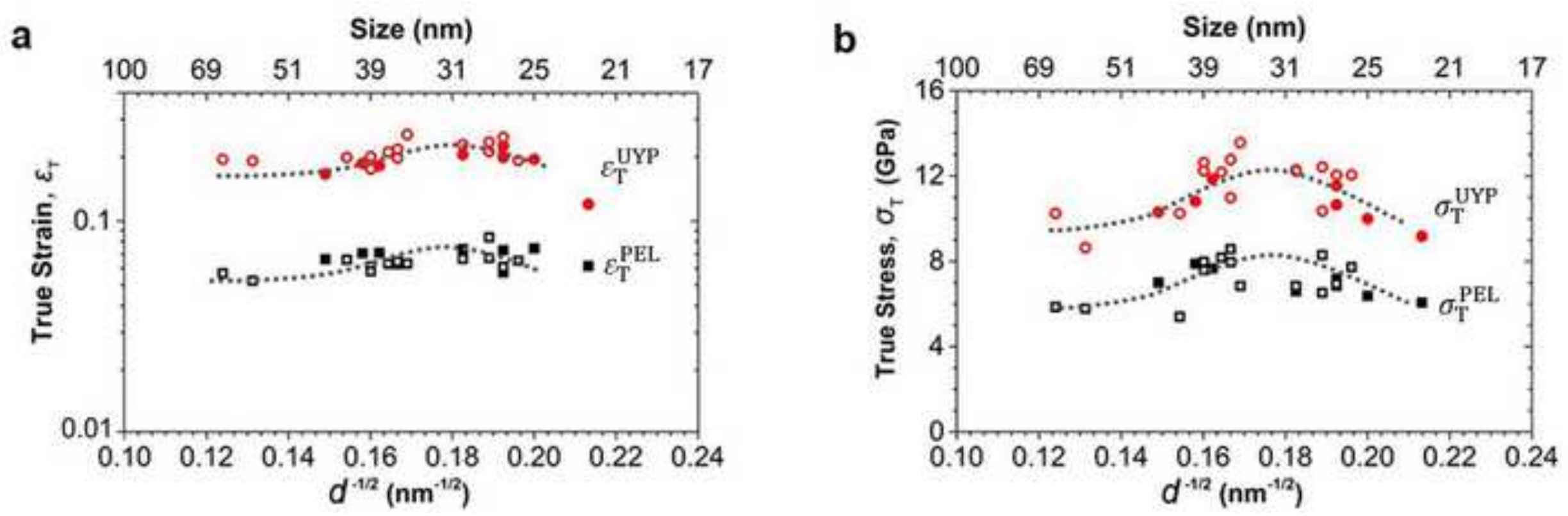
Figure 4
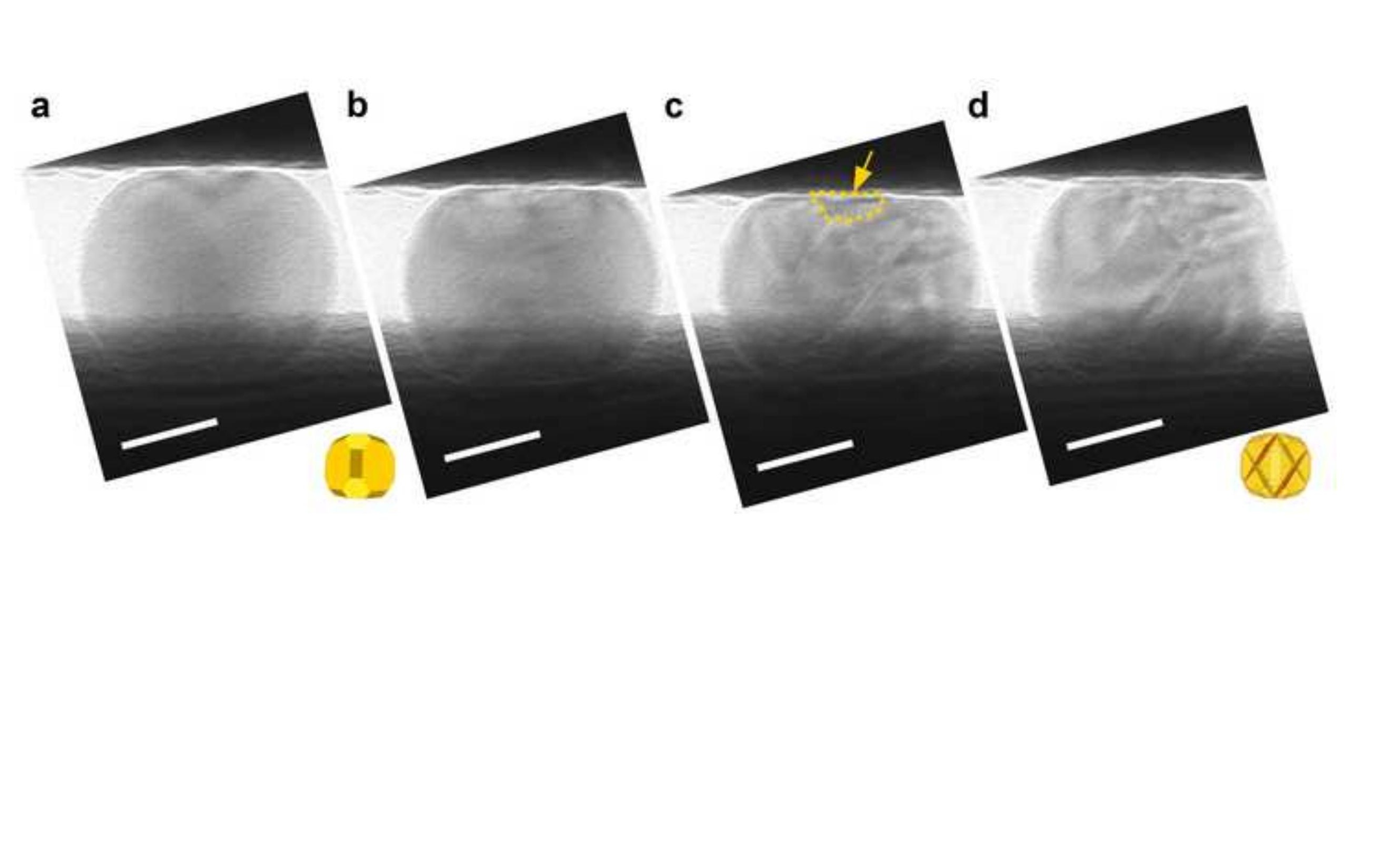

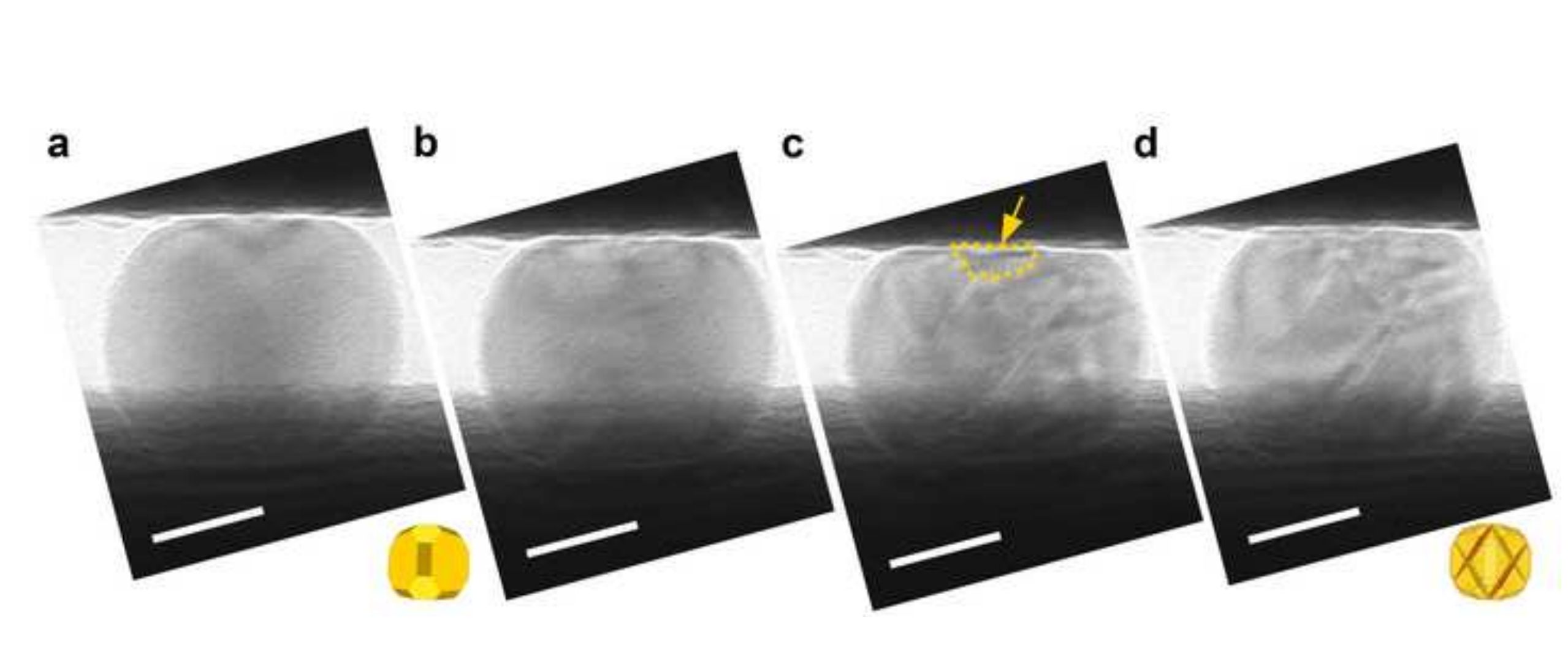

(

4
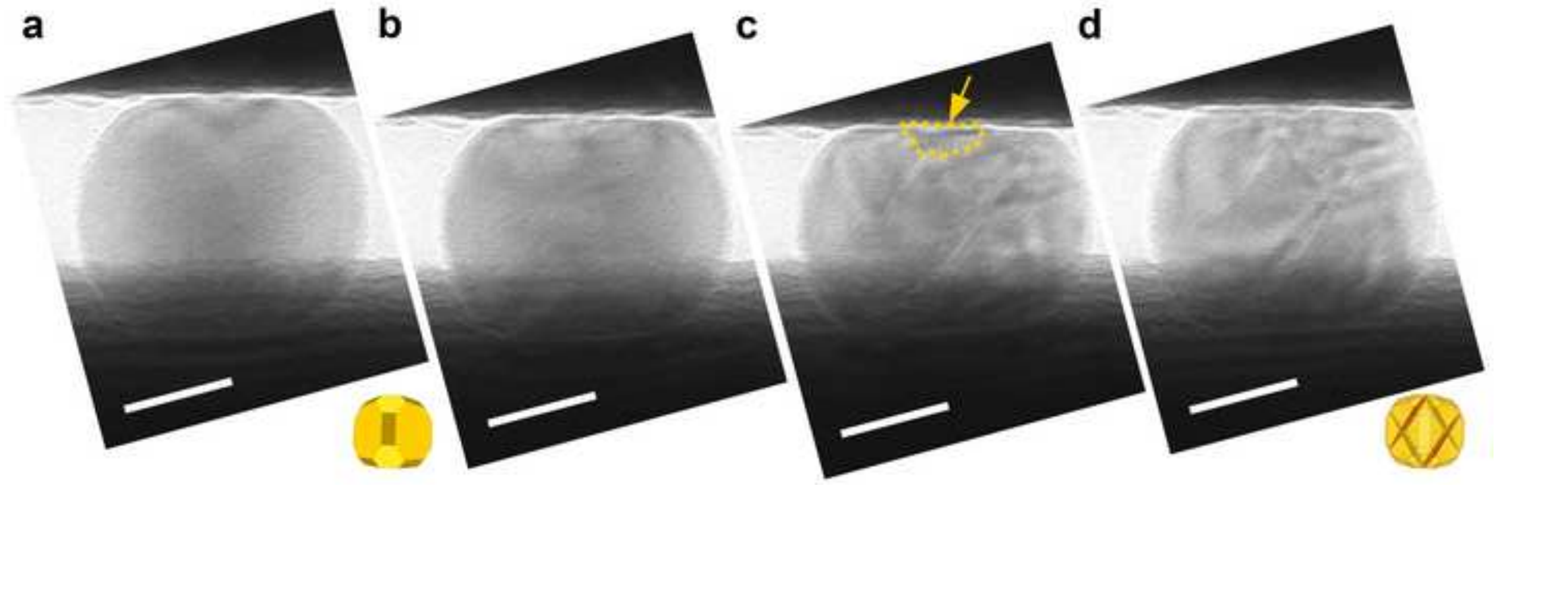
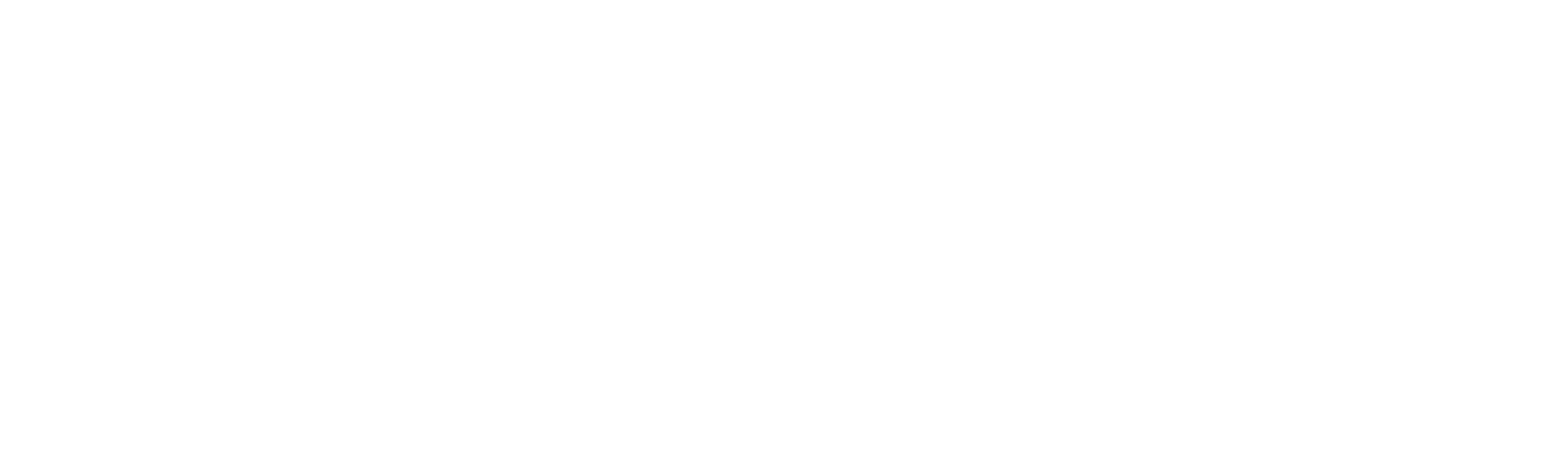

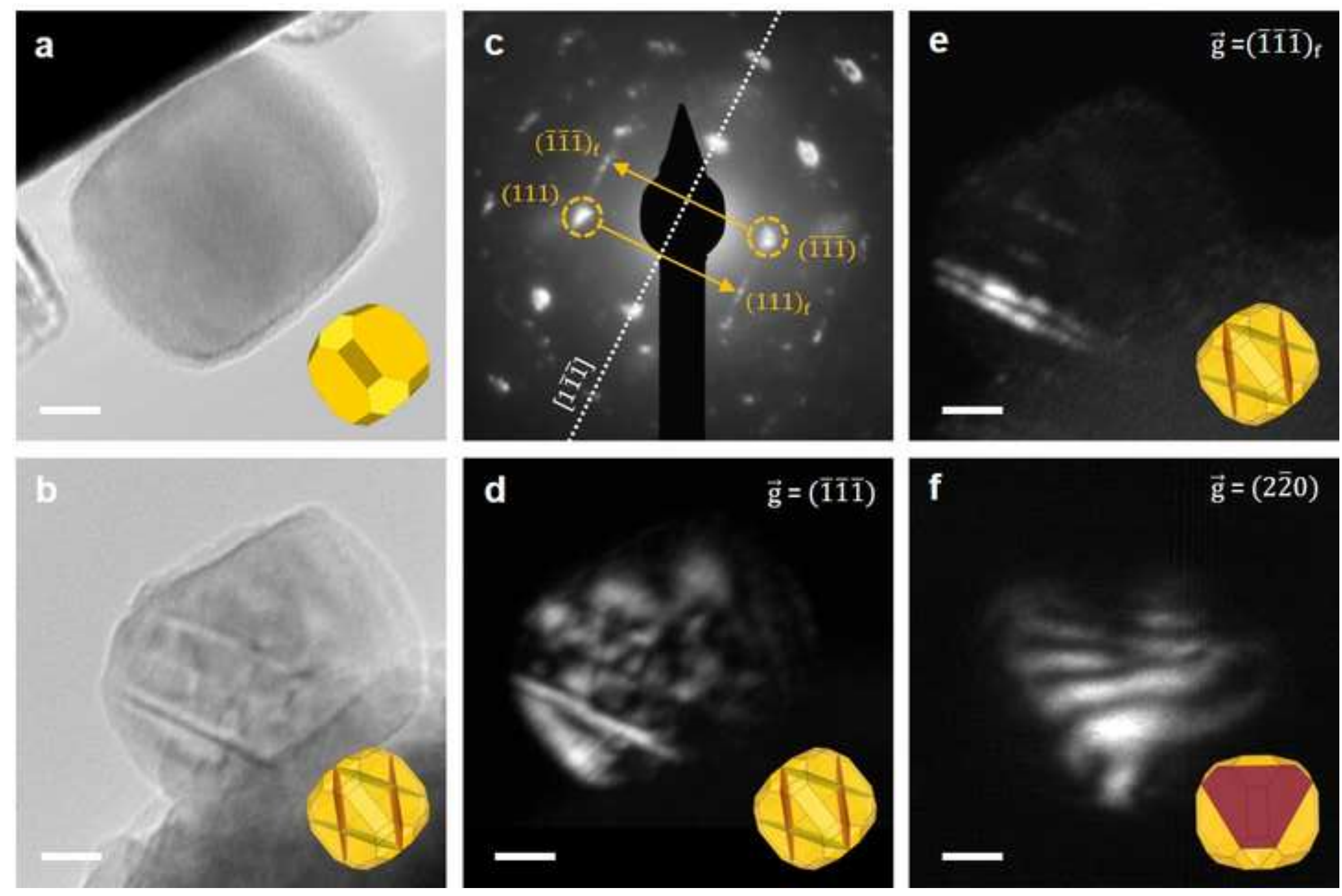

f
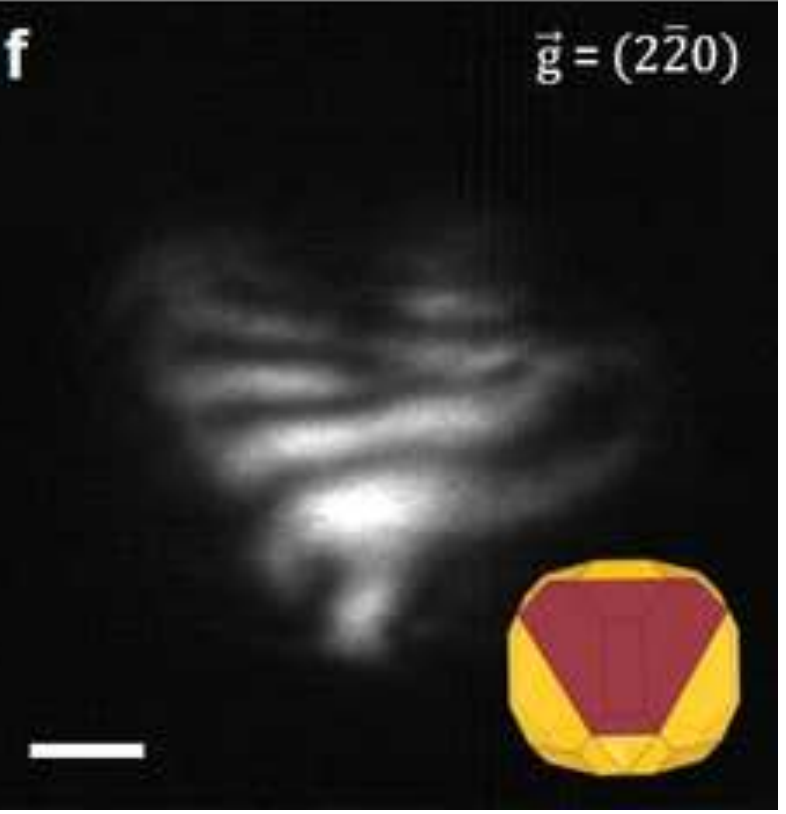

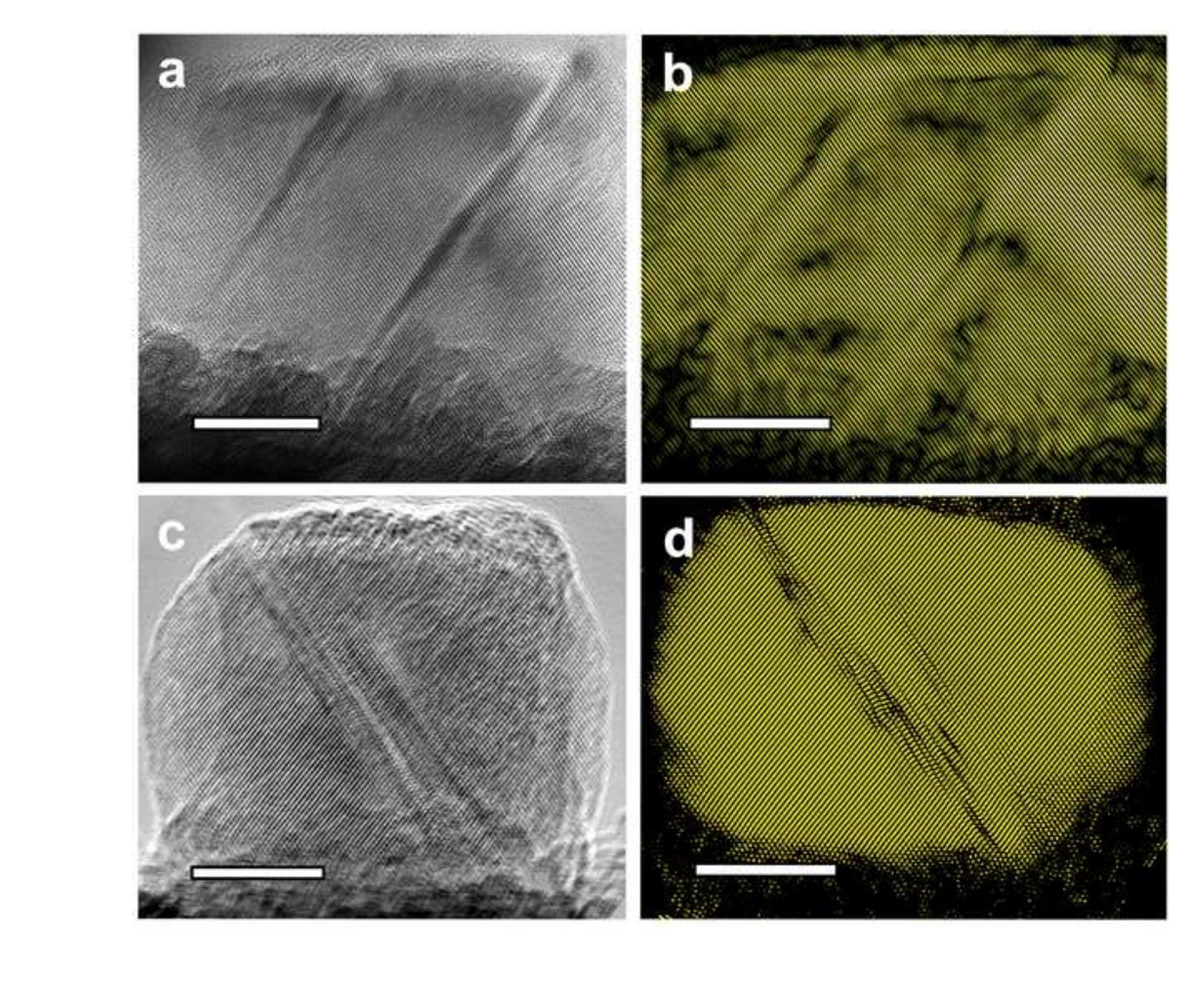


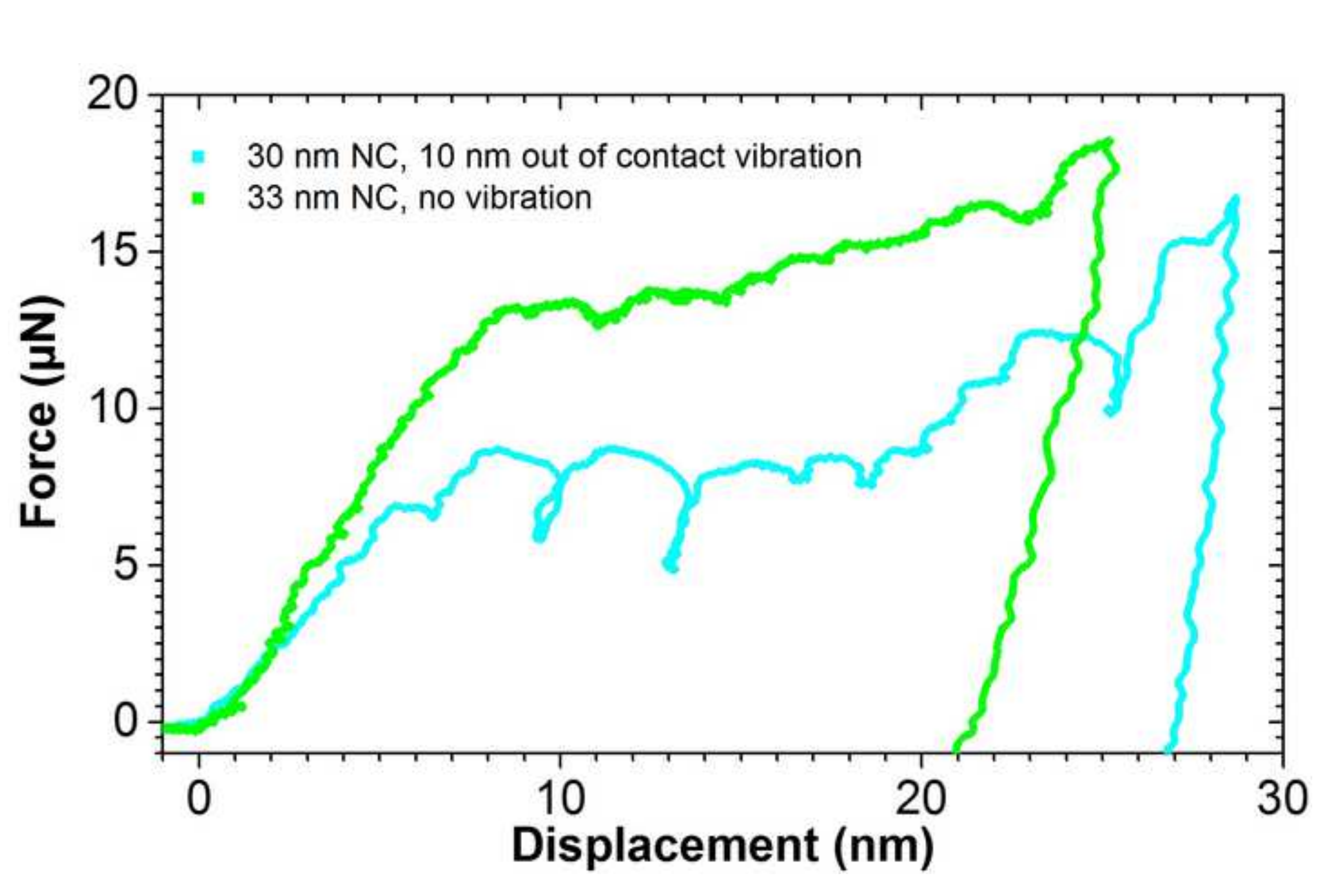


a

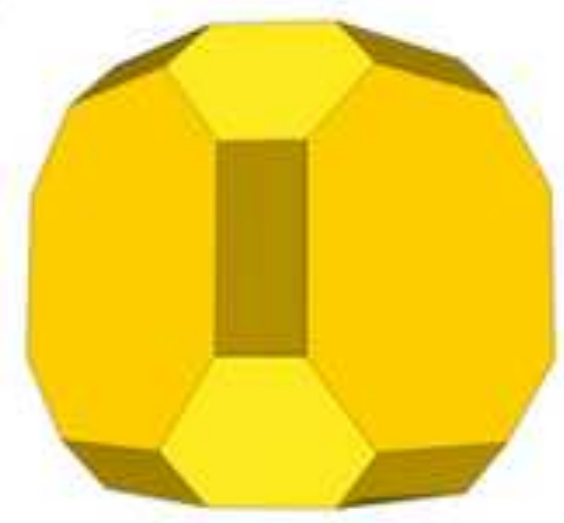

베 $\{111\}$

믈 $\{110\}$

III $\{100\}$

b

$10 \mathrm{~nm}$

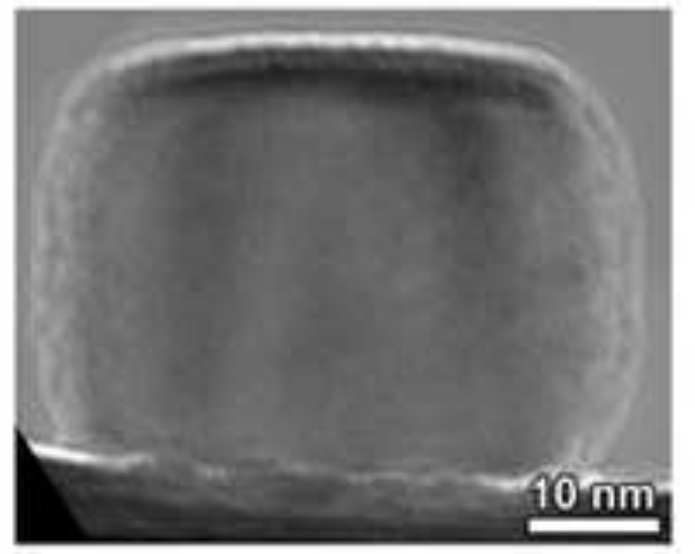

C

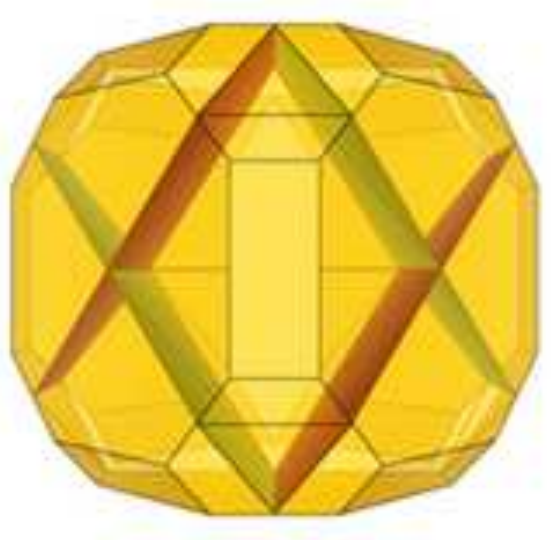

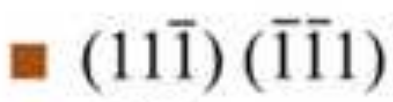

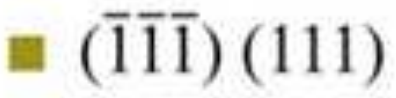

e

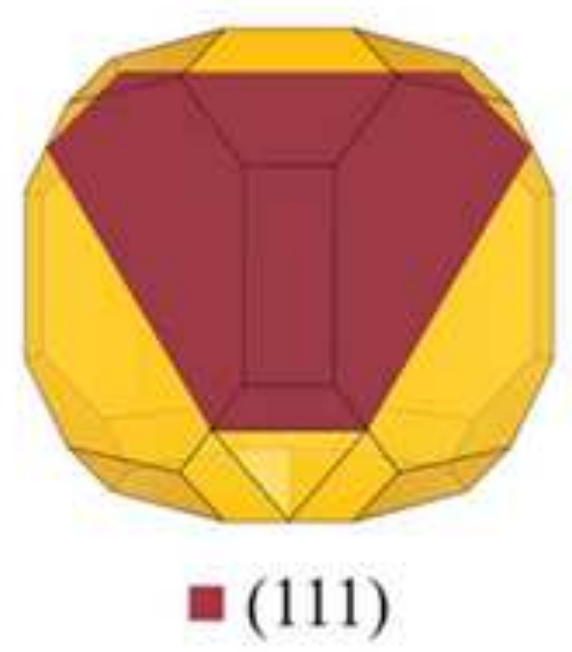

- (111) d c

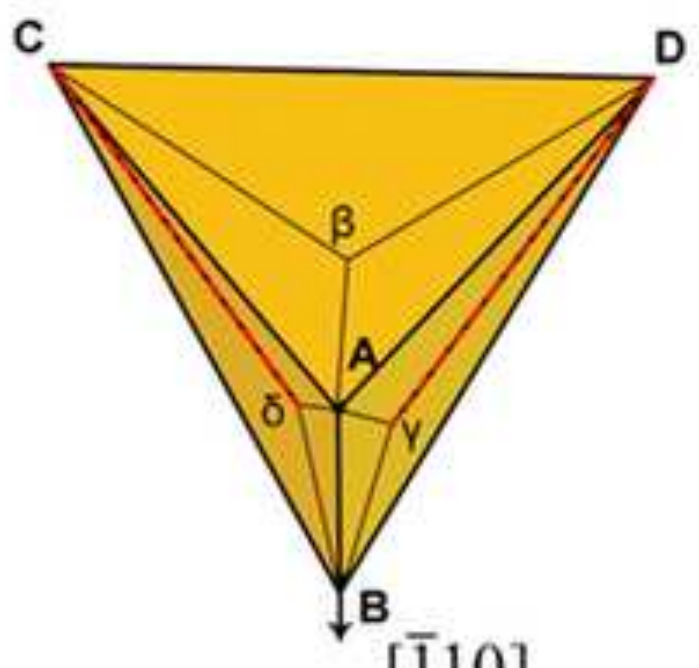

[110]

f

[110]

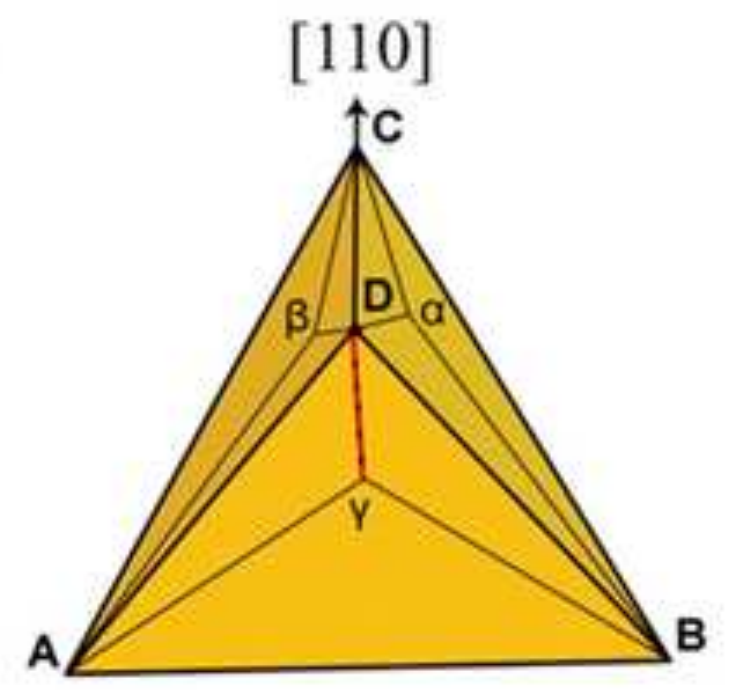


a

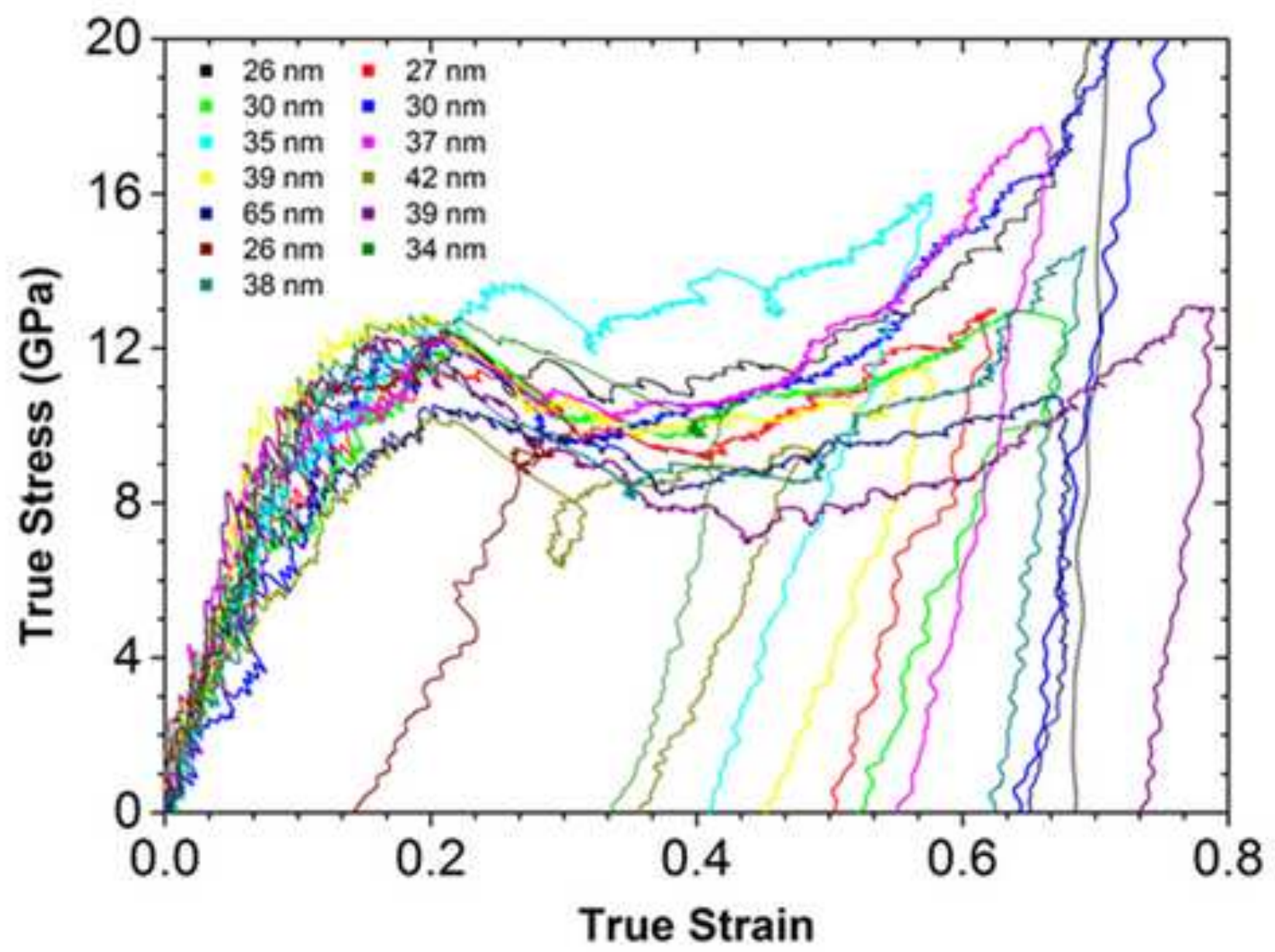

b

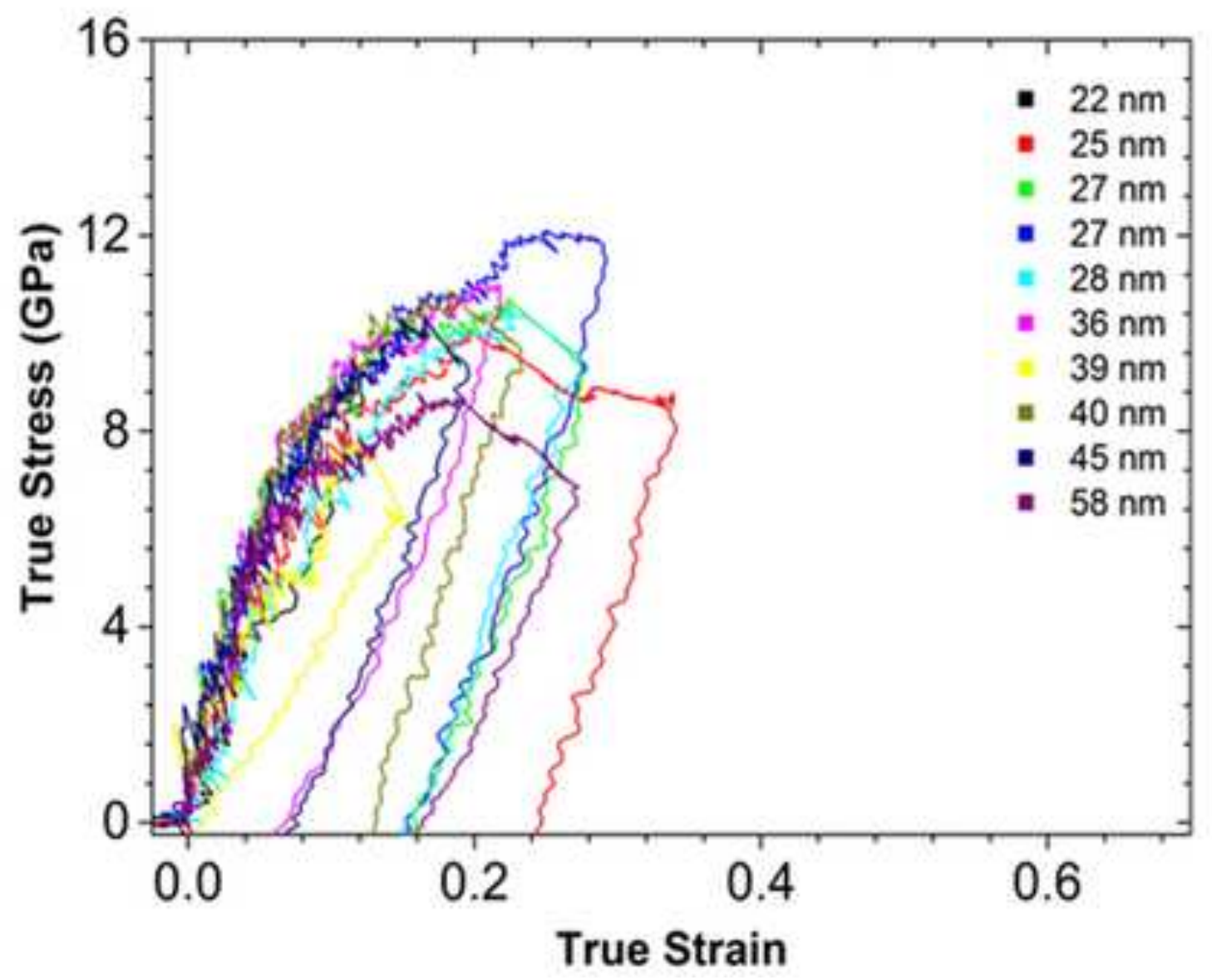



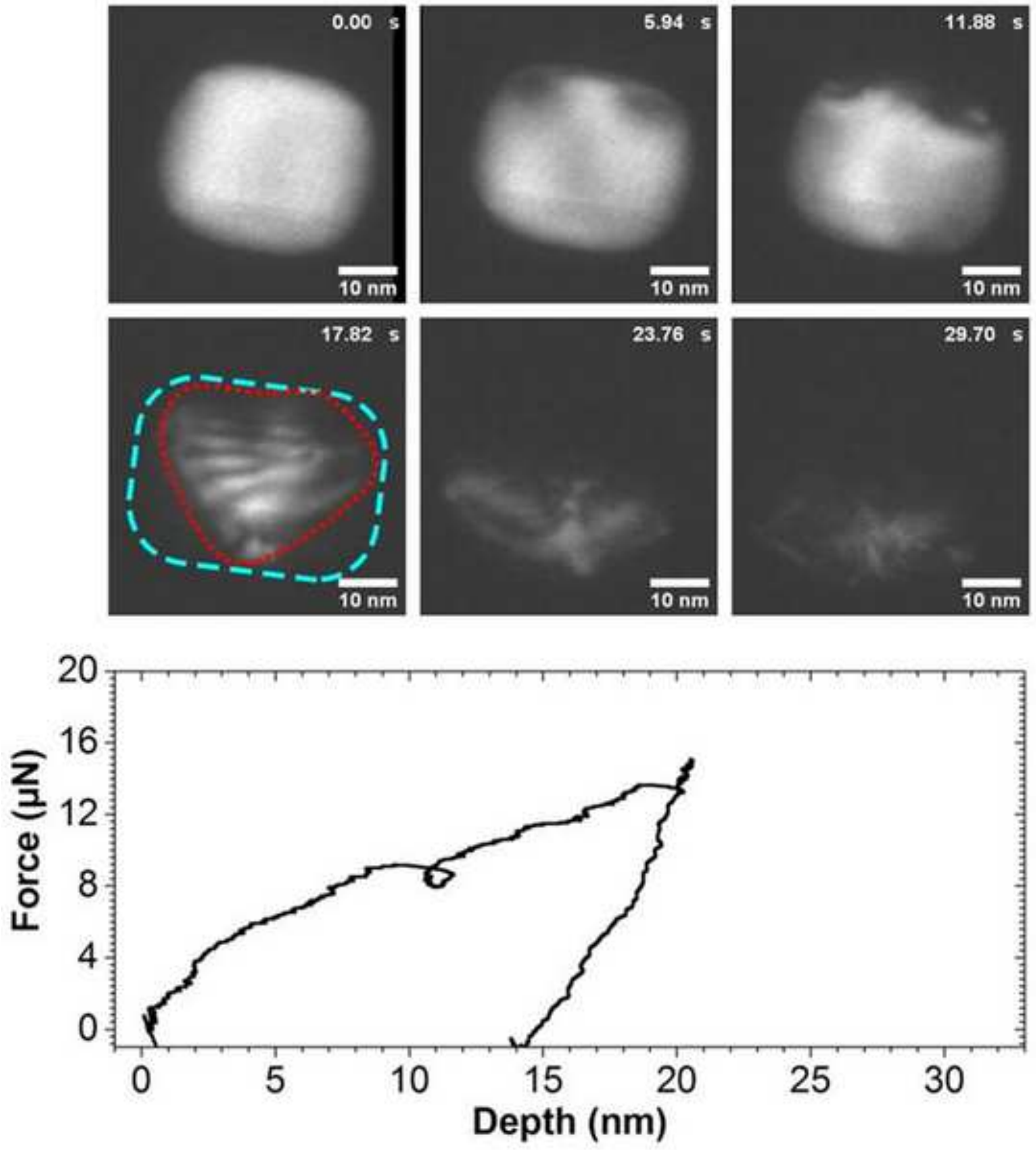
a

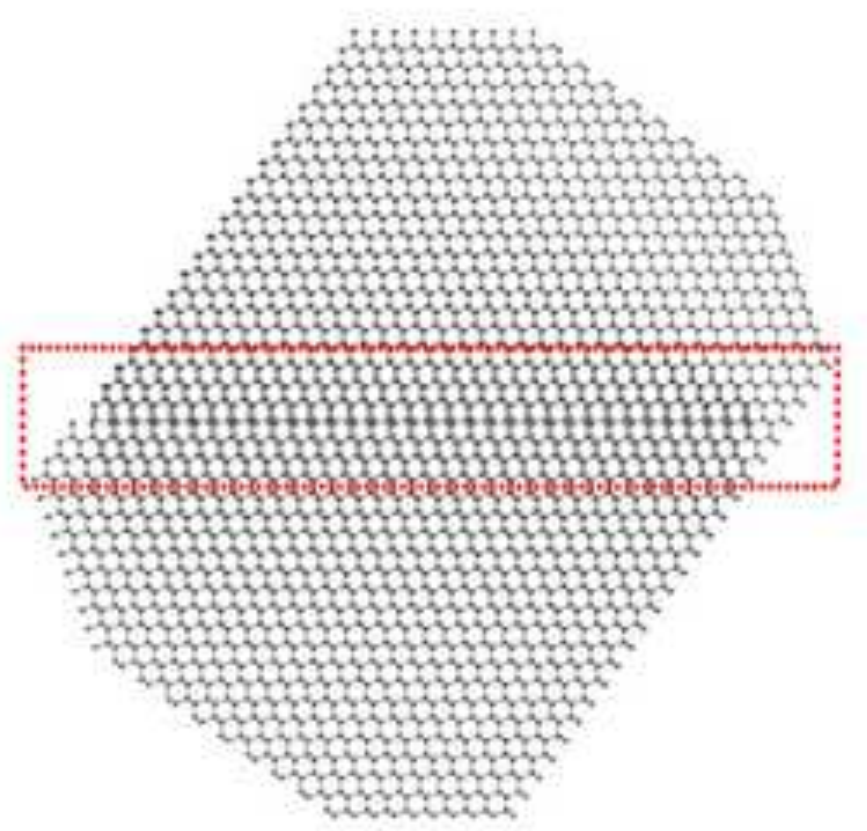

b

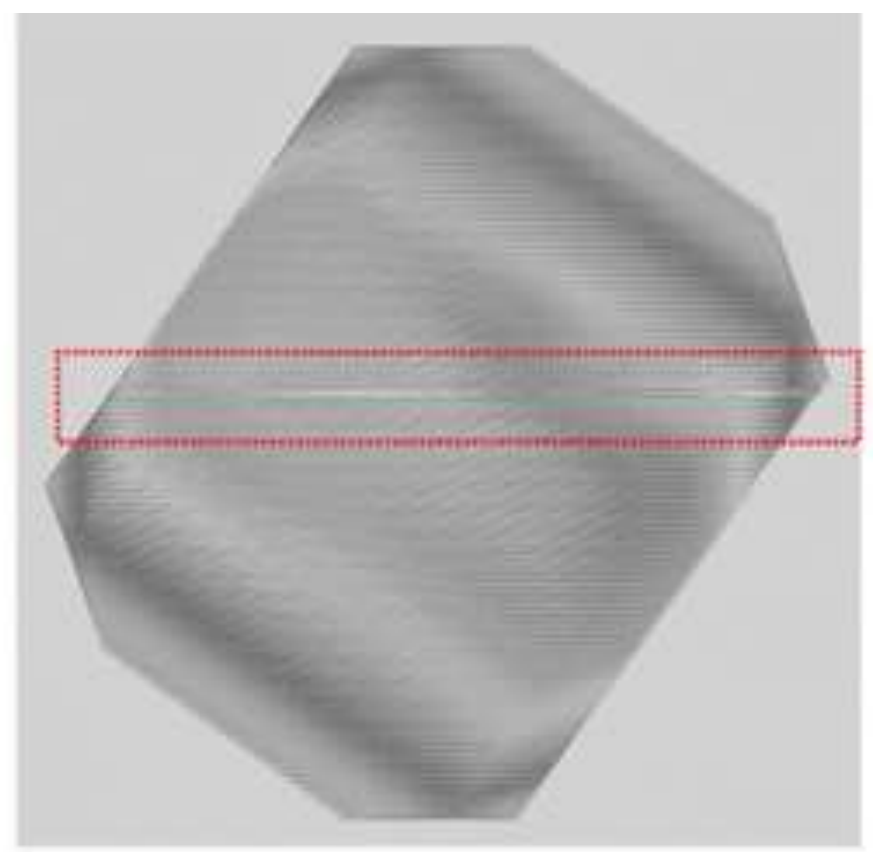

c

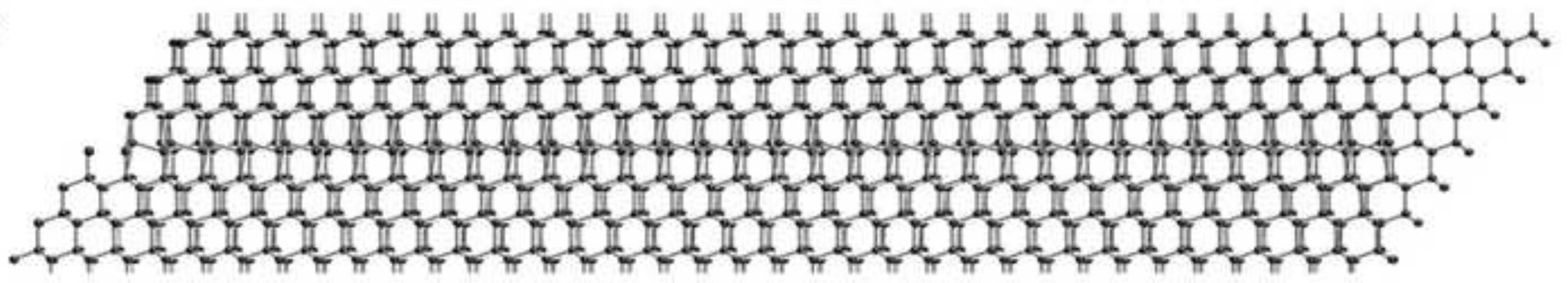

d

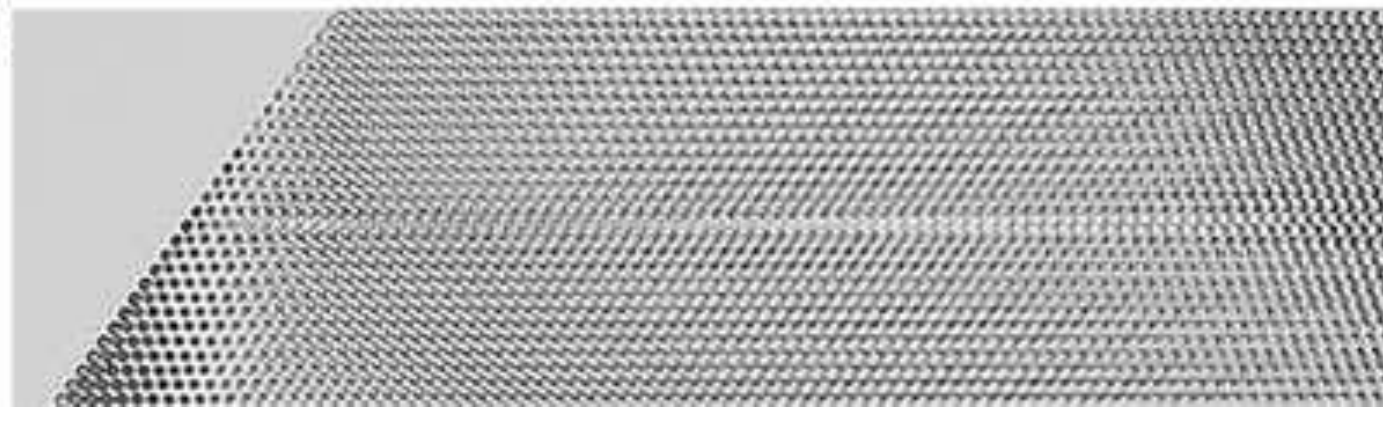


a

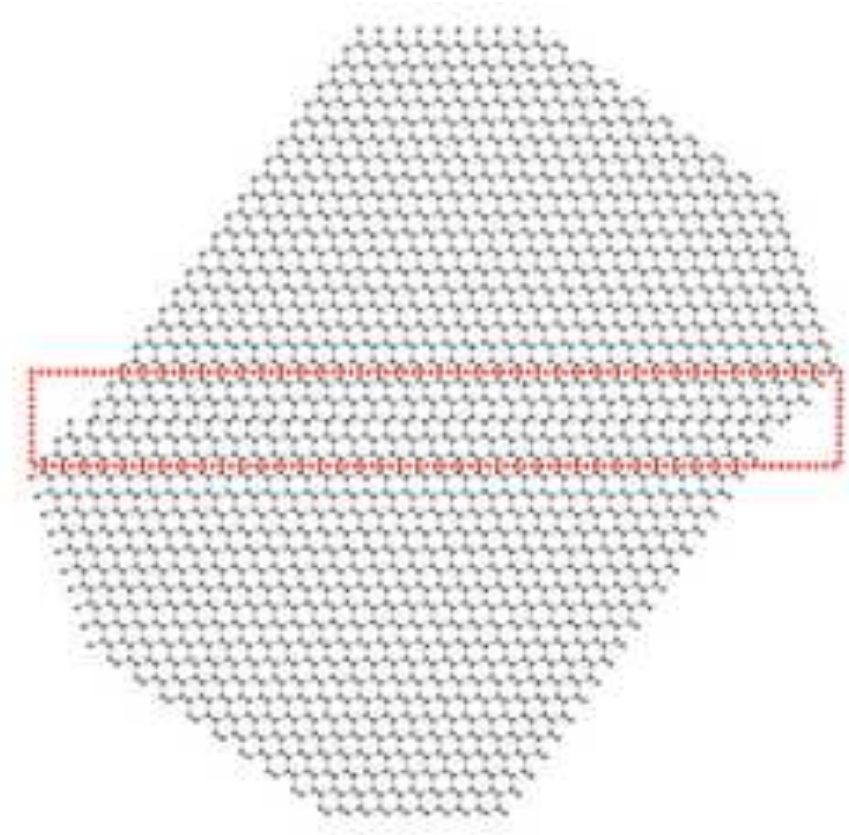

b

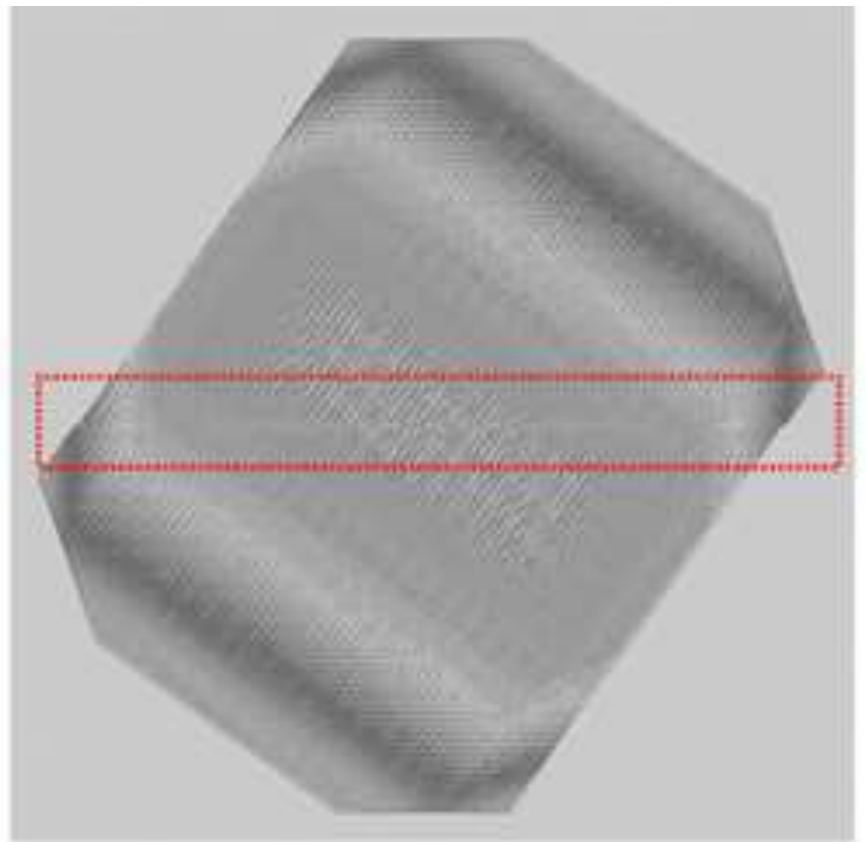

c

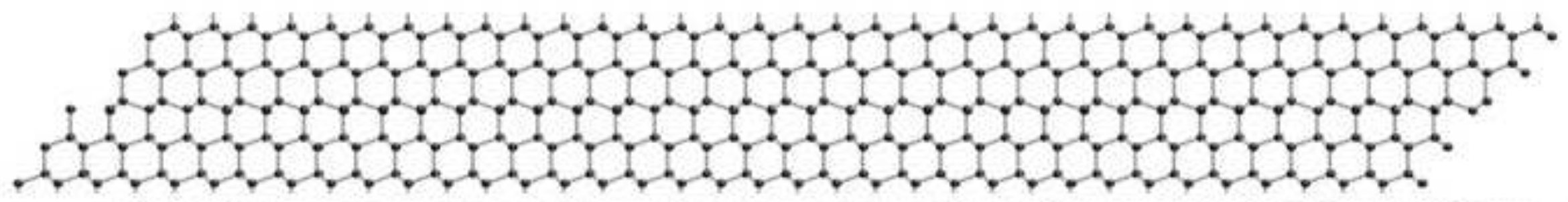

d

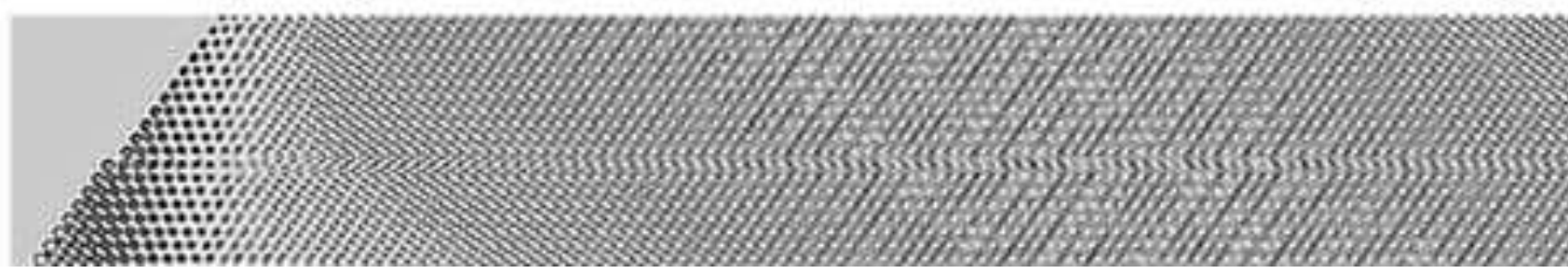


stacking fault

$0^{\circ}$

$1^{\circ}$

$2^{\circ}$

$4^{\circ}$ screw dislocation
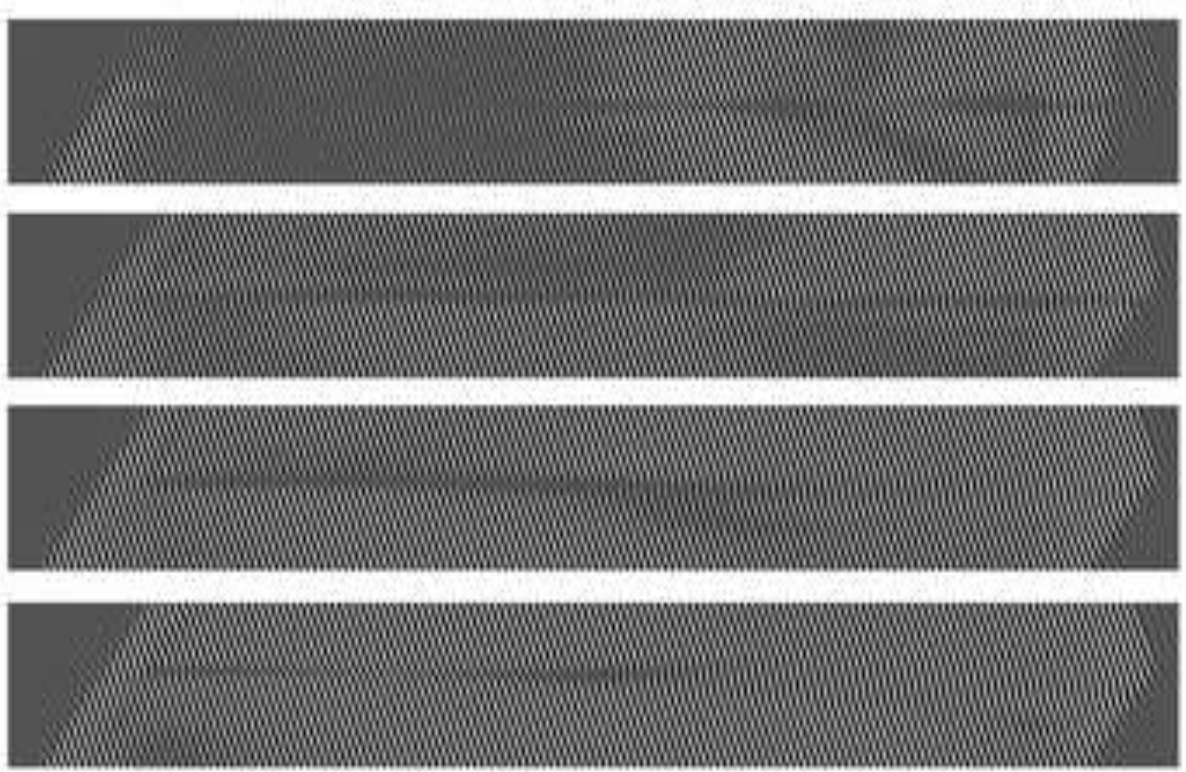
Graphical Abstract

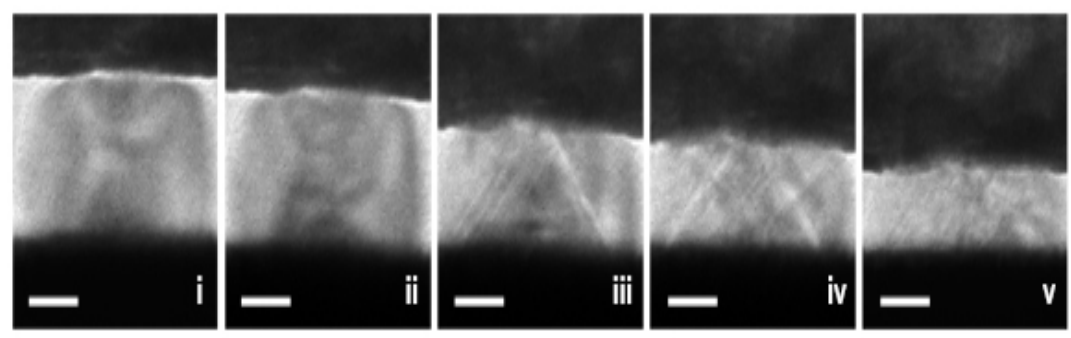

